\title{
Advances in Conceptual Electronic Nanodevices based on 0D and 1D Nanomaterials
}

\author{
Yafei Zhang ${ }^{1}, \quad$ Li Franklin Duan ${ }^{1}$, Yaozhong Zhang ${ }^{1}, \quad$ Jian Wang $^{1}$, Huijuan Geng ${ }^{1}$, Qing Zhang ${ }^{2}$,*
}

(Received 19 October; accepted 4 December 2013; published online 30 December 2013)

\begin{abstract}
Nanoelectronic devices are being extensively developed in these years with a large variety of potential applications. In this article, some recent developments in nanoelectronic devices, including their principles, structures and potential applications are reviewed. As nanodevices work in nanometer dimensions, they consume much less power and function much faster than conventional microelectronic devices. Nanoelectronic devices can operate in different principles so that they can be further grouped into field emission devices, molecular devices, quantum devices, etc. Nanodevices can function as sensors, diodes, transistors, photovoltaic and light emitting devices, etc. Recent advances in both theoretical simulation and fabrication technologies expedite the development process from device design to prototype demonstration. Practical applications with a great market value from nanoelectronic devices are expected in near future.
\end{abstract}

Keywords: Field emission nanodevices; Molecular nanodevices; Quantum nanodevices; Semiconductor nanodevices

Citation: Yafei Zhang, Li Franklin Duan, Yaozhong Zhang, Jian Wang, Huijuan Geng and Qing Zhang, "Advances in Conceptual Electronic Nanodevices based on 0D and 1D Nanomaterials", Nano-Micro Lett. 6(1), 1-19 (2014). http://dx.doi.org/10.5101/nml.v6i1.p1-19

\section{Introduction}

In recent years, with the rapid research development of electronic devices and device miniaturization, nanometer sized electronic devices are in high demand for high performance, small power consumption and fast functionality. As the fabrication ability approaching to nanoscale, both theoretical simulation and design technology have to be developed to capture the development. Many bottle-necks have been resolved in the $R \& D$ stage and reliability of nanodevices has been greatly improved. Technologies and processes suitable for the mass production are also on active stage of development.

Table 1 categories of electronic nanodevices reported so far according to their operating principles and trends. In this paper, their advances and practical ap- plication statues are featured and reviewed.

\section{Field emission nanodevices}

With the recent development of vacuum microelectronics, cold-cathode field-induced electron emission is no long a research topic and nano field emission device (FED) is becoming a practical field. There are two main groups of FEDs: thin-film diamond emission and nanoscale tip emission. Diamond electron emission is based on the fact that the work function of diamond thin films can be zero or even negative. Yet, in order to increase the conductivity, the diamond thin films have to be doped, usually into the p-type. Unfortunately, p-type diamond has a large positive work function. To overcome this obstacle, one has to dope diamond thin films into the n-type. N-type doped

\footnotetext{
${ }^{1}$ Key Laboratory for Thin Film and Microfabrication Technology of the Ministry of Education, Research Institute of Micro/Nano Science and Technology, Shanghai Jiao Tong University, Shanghai, China

${ }^{2}$ School of EEE Nanyang Technological University, Nanyang Technological University, Singapore

*Corresponding author. E-mail: eqzhang@ntu.edu.sg
} 
Nano-Micro Lett. 6(1), 1-19 (2014)/http://dx.doi.org/10.5101/nml.v6i1.p1-19

Table 1 Categories of electronic nanodevices (D: Diode; T: Transistor)

\begin{tabular}{|c|c|c|c|c|c|}
\hline $\begin{array}{l}\text { Application } \\
\text { environment }\end{array}$ & $\begin{array}{l}\text { Functioning } \\
\text { principle }\end{array}$ & Single electron (SE) & Multi-electron (ME) & Junction SJ & $\begin{array}{c}\text { Resistance } \\
\text { sensitive }(\mathrm{RS})\end{array}$ \\
\hline vacuum & $\begin{array}{l}\text { field emission } \\
\text { nanodevices }\end{array}$ & $\begin{array}{l}\text { single nano-tip emis- } \\
\text { sion, single photon de- } \\
\text { vices }\end{array}$ & $\begin{array}{l}\text { multi-nano tip emission, field } \\
\text { emission display D }\end{array}$ & $\begin{array}{l}\text { surface junction } \\
\text { transmitter }\end{array}$ & $\begin{array}{l}\text { dielectric field } \\
\text { emission }\end{array}$ \\
\hline $\begin{array}{l}\text { weak electric } \\
\text { interaction }\end{array}$ & $\begin{array}{l}\text { molecular } \\
\text { nanodevices }\end{array}$ & molecular wires MOS & $\begin{array}{l}\text { molecular rectifier } \mathrm{D} \text {, molecu- } \\
\text { lar tunneling } \mathrm{D} \text {, proton mem- } \\
\text { brane device }\end{array}$ & $\begin{array}{l}\text { molecular } \\
\text { switches, molec- } \\
\text { ular light }\end{array}$ & $\begin{array}{l}\text { molecular wire } \\
\text { sensor, molecu- } \\
\text { lar film sensor }\end{array}$ \\
\hline $\begin{array}{l}\text { low tempera- } \\
\text { ture }\end{array}$ & $\begin{array}{l}\text { quantum } \\
\text { nanodevices }\end{array}$ & $\begin{array}{l}\text { single electron devices, } \\
\text { quantum spin } \mathrm{T}\end{array}$ & $\begin{array}{l}\text { resonant tunneling tubes, nano } \\
\text { spin-D }\end{array}$ & Joseph devices & $\begin{array}{l}\text { Coulombs } \\
\text { blocker }\end{array}$ \\
\hline $\begin{array}{l}\text { room temper- } \\
\text { ature }\end{array}$ & $\begin{array}{l}\text { semiconductor } \\
\text { nanodevices }\end{array}$ & $\begin{array}{l}\text { single nanowire MOS, } \\
\text { particle electrometer }\end{array}$ & $\begin{array}{l}\text { multi-nano wire MOS, thin } \\
\text { films } \mathrm{T} \text {, quantum dot memory } \\
\text { cell }\end{array}$ & $\begin{array}{l}\text { Schottky SJT, } \\
\text { surface plasma }\end{array}$ & $\begin{array}{l}\text { nanowire } \\
\text { sensors, pho- } \\
\text { tovoltaic } \\
\text { nanodevices }\end{array}$ \\
\hline
\end{tabular}

diamond is a technology bottleneck. Discovery of carbon nanotubes (CNTs) in 1991 and other nanowires later make a breakthrough for the sharp nanoscale tip cold cathode electron emission. In 1995, Walt A De Heer in Sweden proposed to use CNTs as an electron emission source [1] and this result has attracted a lot of attention.

CNTs consist of single-wall CNTs (SWNTs) or multiwall CNTs. Their diameters are from 1 up to a few tens of nanometers. Since the diameters are so small, the electric field near the CNT tips is highly concentrated. Comparing to traditional Si cone or Mo cone emitters, emission current of CNTs can be much large under low anode voltages [2-5].

In recent years, most of the efforts are made on electron emissions from a single nanoscale tip and arrayed nanoscale tips, nanoscale surface junction and nanoscale surface dielectric field. Several companies have started their R\&D on FED displays by nanoscale tips. In the IDW'2000 (Workshop in Japan Kobe, Nov. 2000), several electronics companies and research institutes demonstrated their FED display products. Samsung has developed a transistor-like CNT FED display; ITRI in Taiwan has developed reflective CNT FED display. CNT FED display is no longer a research topic since then. Instead, it has been applied in various kinds of equipment. Future trend of the FED display will focus on High Brightness (HB), High Definition (HD), full color and larger size display.

\section{Molecular nanodevices}

Molecular devices have attracted a lot of attention these days and have been becoming a very important topic. In 1974, Aviram and Ratner reported a "molecular diode [6]", in which electrons flowed from donors to acceptors through the $\sigma$-bonds. Later on, "molecular wire" was used to connect the molecular diode to an external electrode. Molecular devices have brought a lot of research interests. Many new models, including molecular switches [7,8], molecular registers [9] have been developed [10-12].

Molecular wire is a key ingredient for molecular nanodevices. The wire should be in the nanoscale and have sufficient conductivity, connecting different joints of various units in a system. Molecular wires can be CNT, DNA, porphyrin and polyphenylene, etc. CNT made of the pure carbon atoms has a very high current density $\left(10^{10} \mathrm{~A} / \mathrm{cm}^{2}\right.$, about $10^{4}$ times of copper wire $[13,14])$ due to its special structure, greatly improves the performance of the molecular device system.

\section{Molecular based nanoswitch}

Nano molecular switch operates in quantum realm and has dual-stable states. The molecular bond can be extended, broken, or rotate by external heat or electronic changes. The switch can be controlled through these external conditions. Electronic switch is the basic electronic control device and has been built on several materials.

Collier et al [15] used rotaxane molecular structure (ring-shape array surrounding a dumb-bell-shaped axis, the ring can rotate and slide along the axis) as the building material for dual-steady state molecular nanoswitches. It was shown that the switches were fully conductive in its reduced-oxidized state yet had high resistance (as an insulator) when in oxidized state. It is able to have two distinct opposite states which make rotaxane molecular structure an ideal material for molecular switch. Afterwards, David et al [16] demonstrated another molecular switch in which exchangeable oxidized and redox properties of Bipy molecules played a key role. When Bipy molecules are in dioxide state, there is no current flowing. When a sufficiently high voltage was applied onto the Bipy molecule through an STM tip, the dioxide state reduces to monoxide state, 
allowing a large current to flow.

Satoshi et al [17] used the reversibility of two states in one molecule to build a switch. They utilized methylisocyanide $\left(\mathrm{CNCH}_{3}, \mathrm{MeNC}\right)$ as a switching molecule and $\mathrm{Pt}$ as an electrode to build the molecular switch. MeNC can be transformed into methylaminocarbyne $\left(\mathrm{CNHCH}_{3}, \mathrm{MeHNC}\right)$ by $\mathrm{H}_{2}$ with $\mathrm{Pt}$ as a catalyst. Meanwhile, MeHNC can be recovered back as MeNC by the aid of tunneled electrons injected by an STM tip. One molecule with two states can achieve two different electronic properties. This is the new idea to make the molecular switches.

Other types of molecules such as fluorescence molecules, magnetic molecules, etc., also have been employed to fabricate molecular computing and logic devices.

\section{Molecular based nano-rectifier}

Rectifier is an important component for discrete circuits and IC chips. Aviram and Ratner first introduced the molecular nano-rectifier in 1974. They used molecular bond to bridge the donor and accepter and build $I$ - $V$ characteristics between two electrodes similar to an ordinary rectifier.

Aviram and Ratner used a self-assembly monolayers (SAMs)-like organic molecules for the devices. These kinds of molecules have a $\mathrm{D}-\sigma$-A bond, where $\mathrm{D}$ is the strong electron donor, A is a strong acceptor and $\sigma$ is the saturated "sigma" bridging compound. $\sigma$ keeps the mechanical strength of the molecules and blocks the exchange of charges between D and A to form a new compound. The key of the molecular rectifier is to ensure that the electron movement between the metal electrodes is via the vacuum tunneling. The charge exchange will create the compound which will then enhance the tunneling effect through the bond. Therefore the barrier of $\sigma$ between A and D should be large enough to avoid the compound forming between $\mathrm{A}$ and D.

Aviram and Ratner used TTF as D, TCNQ as A and bridged them with $\sigma$ bond. The theory is solid yet hard to build device model. Metzger $[18,19]$ proposed a D- $\pi$-A model, replacing the $\sigma$ bond with a non-saturated bond $\pi$. This zwitterionic molecule is abbreviated as C16H33Q-3CNQ, which consists of a body quinoline, bridged $\pi$ bond, $3 \mathrm{CNQ}$ acceptor and ended with a $\mathrm{C}_{16} \mathrm{H}_{33}$. $\mathrm{C} 16 \mathrm{H} 33 \mathrm{Q}-3 \mathrm{CNQ}$ film is very stable, the particles can be uniformly transferred to the metal surface to form a single layer (or multi-layer) molecular film. Molecular C16H33Q-3CNQ $I-V$ curve has typical rectifying properties. However rectification ratio of C16H33Q-3CNQ is relatively low, practicality is not strong.

National Laboratory of Nano-Physical Sciences at University of Science and Technology in China success- fully use a nitrogen atom to replace a carbon atom in Fullerene- $\mathrm{C}_{60}$ and introduce a special donor level in the energy band; this leads to a one-molecular rectifier by single electron tunneling effect [20]. A double barrier tunneling junction (DBTJ) system was built by putting $\mathrm{C}_{59} \mathrm{~N}$ molecule in between a scanning tunneling microscope (STM) tip and SAM. One barrier was in between the STM tip and $\mathrm{C}_{59} \mathrm{~N}$ and the other was in between $\mathrm{SAM}$ and $\mathrm{C}_{59} \mathrm{~N}$. It was suggested that no barrier in between the SAM and Au substrate exists due to the strong bond interaction. In contrast, a barrier in between SAM and $\mathrm{C}_{59} \mathrm{~N}$ could exist due to the weak bonding therefore no charge transfer. Experimental data show that the $I$ - $V$ curve of this DBTJ system exhibited a strong Coulomb blockade effect under certain circumstances. It was shown that various $\mathrm{C}_{59} \mathrm{~N}$ molecules exhibit a significant asymmetry in their rectifying property under different test conditions and similar VF and BV values. It was found that reducing the STM tip and $\mathrm{C}_{59} \mathrm{~N}$ distance led to increase the tunneling current and reduce the BV due to the lower barrier while keeping the VF value unchanged. Although the surfaces of the $\mathrm{C}_{59} \mathrm{~N} / \mathrm{SAM}$ and $\mathrm{C}_{60} / \mathrm{SAM}$ were of the similar properties, the $I$ - $V$ curve of the $\mathrm{C}_{60}$ is symmetric Coulomb blockade curve, while the $I-V$ of SAM is a near-symmetric single junction tunneling characteristics.

\section{Electromechanical molecular nanotransistors}

The basic principle of molecular nanotransistors is to change the molecular structure by external force to achieve the switching function. The external force of molecular transistors can be either mechanical or electrical forces. A typical example is an electromechanical molecular transistor which is an atom relay transistor (ART) [21]. The advantage of ART is very small size (dimensions about $100 \mathrm{~nm}$ ). Its switch control is realized through the conductivity change due to the mechanical motion of the atoms along the atomic line. In ART, there is a non-fixed atom on the substrate and can be moved back and forth across the transistor. When switching atom moves to the center on the line by gate control, an "ON" state is achieved. When the atom leaves atomic lines, the transistor is then in the "OFF" state. The intrinsic oscillation frequency of the atom determines the switching speed; different atoms have different speeds. For carbon atom, the switching speed can reach $100 \mathrm{THz}$. It is very important to choose appropriate atoms that could make ART work at very high speed. Another advantage of ARTs is that the devices consume very low power. The energy is consumed by the friction between individual atoms and the substrate. In contrast to ART's obvious advantages, its disadvantages are also prominent. The energy required to emit an ART atom from the atomic line on substrate 
plane is too small, at which point the switch would be distorted. In order to control ART properly, low temperature environment is required.

Another example of electromechanical molecular nanotransistor is supra-molecular atom transistor (SMART) [22]. Super-molecules and atoms can be rotated by the gate voltages. The rotational position controls the conductance of the atomic line. In SMART, the switch atom moves in and out from atomic line by the gate electric field. When the switching atom rotates into the atomic line, atomic line is conductive; when the switch atom is out of atomic line, atomic line loses conductivity. Atomic radicals located on the rotational isomers group can prevent the rotation of a substitution group due to heat. Rotation switch group to prevent atomic emission is very effective, better than ART. But the switch of SMART is achieved by the co-rotation of all the three groups, leading a relatively lower reliability. If the number of control groups drops to two, it will have more practical value. Cyclohexane is a good example that it can be bent into two different forms. The gate voltage changes the structure of cyclohexane, thus affecting the conductivity of atomic line. By comparison, a group-controlled rotary switch is significantly slower than atomic motion control switch, but the controllability is much better than atomic switch. SMART energy consumption is mainly on overcoming the weak attractive van der Waals interaction or hydrogen bond, so power consumption is very low.

Electromachanical molecular transistors can also be made using $\mathrm{C}_{60}$. $\mathrm{C}_{60}$ conductivity can be adjusted by the STM probe pressure [23-25] to achieve switching functions. According to the experimental observations, $\mathrm{C}_{60}$ molecule transistor exhibits step-like current jumps under higher bias. The conductivity was strongly prohibited near zero bias voltage and this voltage range can be adjusted by gate voltage.

\section{Molecular single electron transistor}

Molecular single electron transistor, MOSET [26] is of the T-shaped structure: the top is a drain and a source, the bottom is the gate, wherein the drain, source, and gate electrodes are made of a conductive molecules. Other components such as single-electron tunneling junction barrier and the gate insulating layer are made of insulating molecules. The Coulomb island between the source and drain electrodes is constituted by the conductive molecules. Such device structure can work at the speed over $1 \mathrm{THz}$.

\section{Molecular light emitting nanodevices}

Molecular light-emitting nanodevice (single molecule light emitter device) is composed of an electron transport layer, a light emitting layer, and a hole transport layer together with an electrode connector, wherein the electron transport layer, light emitting layer and hole transport layer are the inner molecular layers where carriers can transport, recombine and emit light. The electrode connector is near the edge of the molecular layer in order to connect each molecular layer, formed by the reaction of mercaptans and carbon. Since light emitting molecules are the only carriers in the system and no other carrier channel, the injection efficiency, carrier transport efficiency and carrier recombination efficiency can reach almost $100 \%$ in this type of singlecarrier light-emitting device. Another feature of this device is by selecting appropriate molecular structure of LUMO-HOMO, one can get the light of any wavelength. As potential barrier between electrodes and intermolecular layers of the emitting device is small, working voltage and power consumption are low so that overheating effect and hot carrier generation can also be avoided. Molecular light emitting devices have excellent flexibility and a wide range of applications.

Although molecular nanodevice technology has many great advantages and large room for further development, it still faces many challenges. Molecular nanodevices can only work in the weak state. Thus, their stability and durability are poor. Most of the devices are still in the laboratory research stage. Therefore, there is a long journey before the devices are pushed to the markets.

\section{Quantum nanodevices}

Quantum nanodevices cover electronic nanodevices, optoelectronic devices, integrated circuits, etc., which are work based upon various quantum effects, such as quantum size effect, quantum tunneling effect, Coulomb blockade effect, nonlinear optical effects and quantum information processing. Design and production of solid state quantum nanodevices are currently the most active research direction in nanoscale physics and electronics. Since most nanostructures are smaller than the de Broglie wavelength of electrons $(50 \mathrm{~nm})$, the electron quantum fluctuations behavior appears. One practical example is the low temperature superconducting quantum device - the Josephson device. Single electron quantum devices, tunnel diodes, spintronic nanodevices, quantum dot single-photon source and quantum dot laser belong to this category.

Single-electron transistors have a very narrow conductive channel in which two potential barriers are artificially introduced. The region between two barriers is the partition section (or Coulomb island). At very low temperatures, the gate voltage can shift the energy levels in the Coulomb island and change number of electrons in this region, causing the conductance periodically to oscillate once which is equivalent to the device go through "ON" and "OFF" cycle once. This process 
is significant for the understanding of electron transport properties in nanostructures and single-particle physics. This study is also likely to lead further practical applications.

Resonant tunneling diode (RTDs) [27] is a twoterminal quantum nanodevice consisting of quantum wells and quantum barriers. The materials are mostly III-V compound semiconductor hetero-structures. As quantum well is only a few nanometers thick, the energy levels are widely spaced in quantum well due to quantum confinement. Generally RTDs work only with one electron energy level; its differential resistance can be either positive or negative. RTD is faster than then high electron mobility transistor (HEMT) - the oscillation frequency has reached $700 \mathrm{GHZ}$. Most RTDs use III-V compound semiconductors such as GaAs and InP technology, and are mainly for military and defense use.

Spintronic nanodevices [28] depend on injection, transfer, operation, zoom-in and read-out of spin polarization in the device structures, in order to achieve integration of logic operation, non-volatile random access memory and communication functions in a single chip - the next new generation of multi-chip. Multilogic device is achieved by the tunnel effect in the ultrafine structure, large-capacity memory by quantum box structure, high-speed switching devices and sensors by the use of coherent electron wave interference, diffraction and reflection, and quantum boxes/quantum dot laser are achieved by changing density of states. As the spin-based quantum bit has much longer quantum coherence time than that of electric charge, it will hopefully become a scalable solid-state quantum-bitthe next new generation of versatile, high-performance, ultra-high speed and low power spin quantum nanodevice.

\section{Semiconductor nanodevices}

According to famous Moore's law [29], in the next few years integration density and computing power will continue increasing and cost per bit will continue decreasing. The fundamental requirement is to keep the device basic functions unchanged when the device is getting smaller. This is achieved by the so-called scaling-down principle: to keep the same internal electric field during the shrinking progress: shrink the voltage and dimensions by a factor $\mathrm{K}$ and increase doping density by a factor of $\mathrm{K}$. The scaling down rule will (1) Reduce short channel effect to maintain the channel characteristics; (2) Increase the current density by K times; (3) Reduce delay time by a factor of K; (4) Reduce power consumption by a factor of K2. Yet, when the device continues to shrink down, we need to consider (1) to reduce the heating effect and improve heat dissipation of the devices, (2) to reduce statistical fluctuations in any multi-body systems for device performance consistency, and (3) the quantum behaviors of electrons when the device operates in quantum range such as the tunneling, $0 \mathrm{D}$ and $1 \mathrm{D}$ effect in quantum dot and quantum wire. In the following some most-recent progresses on electronic nanodevices are reviewed.

\section{Metal oxide semiconductor (MOS) nanodevices}

Metal oxide semiconductor field effect transistors (MOSFETs) devices are a kind of widely used fieldeffect transistors in analog and digital circuits for several decades. The size of nanoscale MOS devices continues shrinking, while their performances are enhanced: (a) The area of MOSFETs is getting smaller so that number of chips per wafer increases and the cost per chip decreases; (b) The equivalent channel resistance is reduced, allowing more current flowing through; (c) The equivalent gate capacitance is also reduced. The negative impacts of MOSFET shrinking include: (a) More difficult to shut down the FETs; (b) Significant increase in power consumption due to its sub-threshold leakage; (c) Increase in parasitic capacitance from chip wire connection; (d) Increase in chip heating; (e) Increase in gate oxide leakage current. Due to small dimensions, the process control becomes even more challenging.

With improved integration and the shrinking of microelectronic devices on a chip, new nanomaterials and new electronic solid-state nanodevices could be more suitable for next generation electronic applications. Nanodevices, due to their critical dimensions down to the nanometer range (such as cross-sectional dimension of unipolar device channel, barrier/potential well layer thickness of bipolar junction scale tunneling devices, nanoscale island-to-island devices, etc.) which are close to Bohr radius, a few physical characteristics such as non-equilibrium transport (carrier velocity overshoot), atomic-level fluctuations and quantum effects (quantum confinement, quantum transport), etc. [30] become significant and dominate. Currently, mainstream nanometer semiconductor devices are: Schottky barrier FET [31], dual-gate FET, and surrounding gate FET structure $[32,33]$. Specifically, the coaxial surround-gate structure built by nanomaterial assembly technology can reduce drain-induced barrier lowering (DIBL) effect and the traditional short-channel effects of MOSFET while achieving a high transconductance and a low power consumption.

\section{Semiconductor carbon nanotube field effect transistor (CNTFET)}

\section{Advantages of CNTFETs}

CNT [34-38] has superior electronic properties such as: (1) Distinctly different electrical characteristics 
from Si: carrier transport is one-dimensional and scattering phase space on carrier is smaller (resulting in ballistic transport and the lower power consumption); (2) Full chemical bond for C atom-no dangling bond as $\mathrm{Si}$ which requires passivation. This means that $\mathrm{CNT}$ does not require $\mathrm{SiO}_{2}$ as an insulator. Thus, high dielectric constant or crystal can be used to form insulator in the three-dimensional structure; (3) Strong covalent bonds in CNT enhance its mechanical strength, thermal stability and resistance to electro-migration. CNT can withstand up to a current density of $10^{9}$ $\mathrm{A} / \mathrm{cm}^{2}$; (4) The connection of active devices (transistors) can be achieved either by semiconductor-like or metal-like CNTs. In addition, the semiconducting CNT has a theoretical maximum electron mobility greater than $100000 \mathrm{~cm}^{2} /$ Vs at room temperature, exceeding all the known semiconductors so far. Experimentally, field-effect mobility of $79000 \mathrm{~cm}^{2} / \mathrm{Vs}$ at $300 \mathrm{~K}$ is observed, in comparison to less than $1000 \mathrm{~cm}^{2} /$ Vs fieldeffect mobility achieved in silicon MOSFETs [39].

\section{Recent progress in CNTFETs}

In 1998, Tans, etc. [40] made the first CNTFET. As shown in Fig. 1, a single semiconducting SWNT is connected to a pair of $\mathrm{Pt}$ electrodes (the source and drain) which are formed on $300 \mathrm{~nm}$-thick thermal oxide $\mathrm{SiO}_{2}$ capped highly doped silicon substrate (the gate). By scanning gate voltage, the IV feature is very similar to the conventional MOSFET. The working mechanism of CNT is dominated by the Schottky barrier between the metal electrode and CNT. Success of CNTFETs marks a big progress on CNT-based electronic devices.

In order to improve the performance of CNTFETs, scientists have put their efforts in lower contact resistance, better control of the gate electric field on the CNT channels and optimizing the structure design.

Y. F. Zhang's group in Shanghai Jiao Tong University [41] has developed disperse and oriented SWNT arrays for multi-channel CNTFETs (shown in Fig. 2).
In the multi-channel CNTFET fabrication, alternating electric $(\mathrm{AC})$ field was used to arrange the SWNT array. By using the alternating electric field in twodimensional electrophoresis technique, they arrange the functional prettified SWNT connection, making it distributed along the source and drain electrodes in parallel, and then use electric current to selectively burn away metallic SWNTs and leaving semiconducting SWNTs across the $\mathrm{S}$ and D pads.

Grafted octadecylamine (ODA) on SWNT will not form a good electrical contact between the electrode and SWNT. By making use of ultraviolet (UV) irradiation, grafted ODA can be evaporated, thus effectively improving the contact between the electrode and SWNT

Multi-channel CNTFETs greatly improve not only the output current and transconductance, but also other key device performances which are much better than single-channel CNTFETs, making the multichannel CNTFETs a potential future candidate of FETs.

In addition, atomic force microscopy (AFM) probe can be used to move SWNTs along the channel to connect the source and drain pads, thereby controlling the SWNT channel number. Thus, via AC DEP technology and AFM probe manipulation technique, we could achieve the different numbers of SWNTs between a same pair of source and drain electrodes.

When the density of SWNT array was not very high, transconductance of multi-channel CNTFET was approximately proportional to the number of the SWNTs. This allows the control of CNTFET transconductance by adjusting SWNT numbers in the channel to accommodate the needs of different applications.

In 2013 Stanford's group [42] has developed a carbon nanotube mini computer. They make the aligned CNT wires on a quartz substrate and then transfer the CNTs onto the silicon wafer with the already-patterned backgates using $\mathrm{Al}_{2} \mathrm{O}_{3}$ as a high-K dielectric. Then

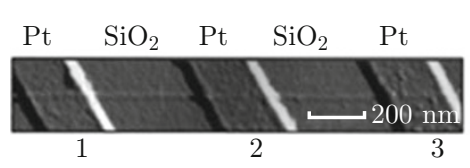

(a)

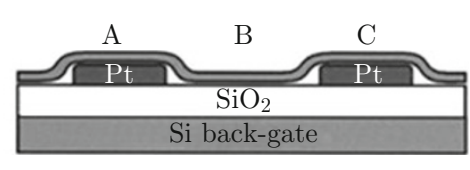

(b)

Fig. 1 (a) CNT connected to three Pt electrodes; (b) a schematic of CNTFET.
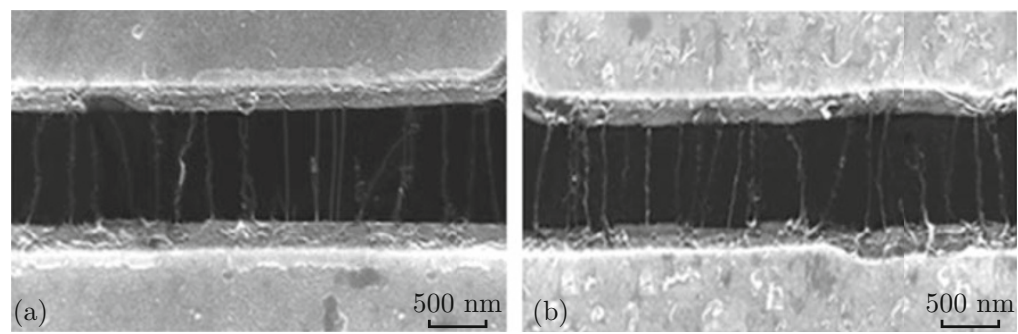

Fig. 2 SEM image of SWCNT solvent between the source and drain electrodes in modified alternating electric field. 

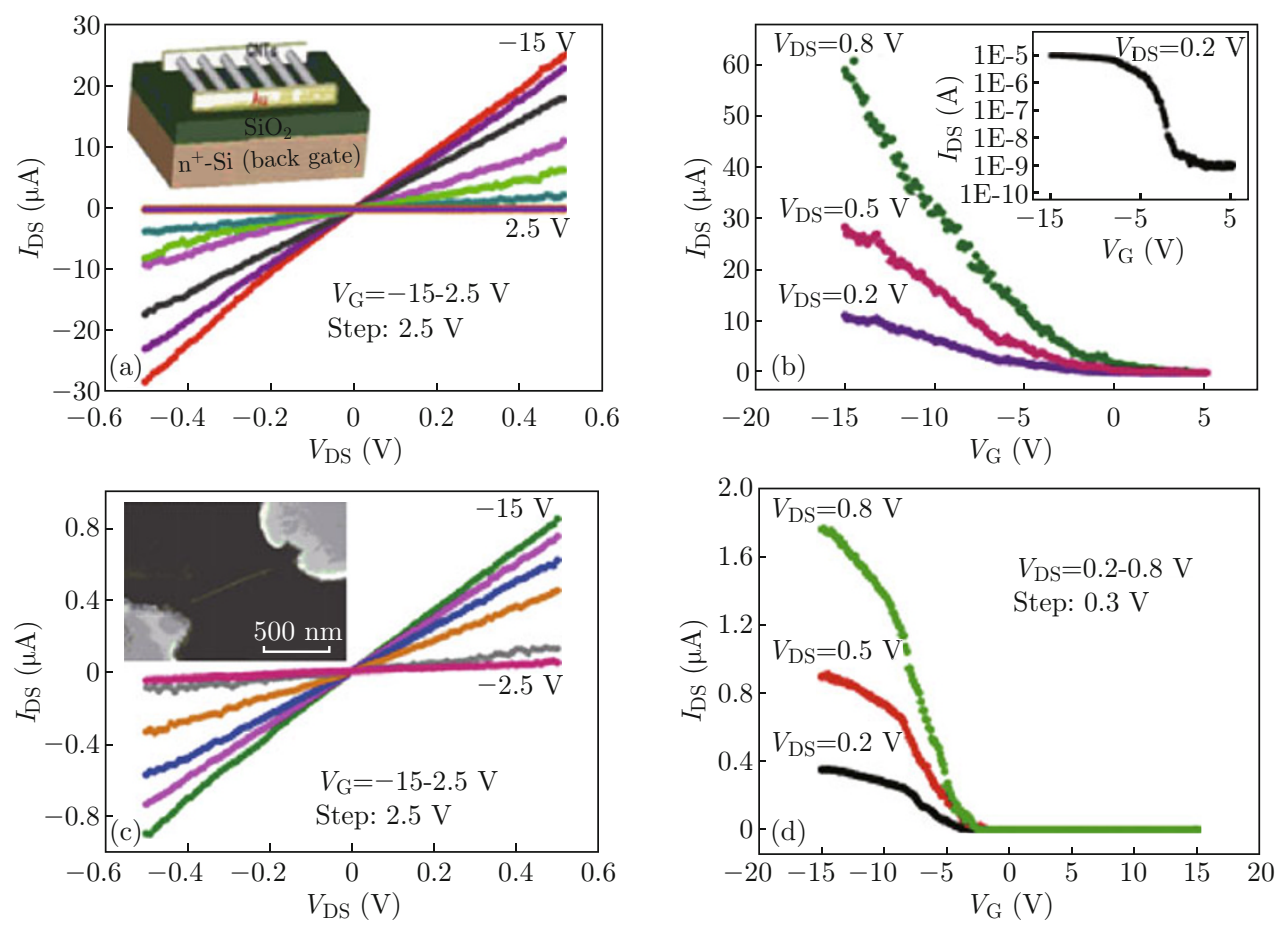

Fig. $3 \quad I-V$ characteristics of multiple-channel and single-channel CNTFET.

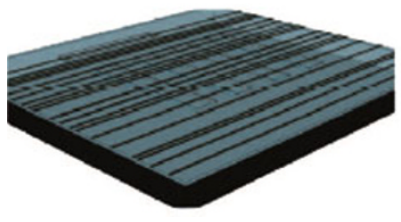

(a)

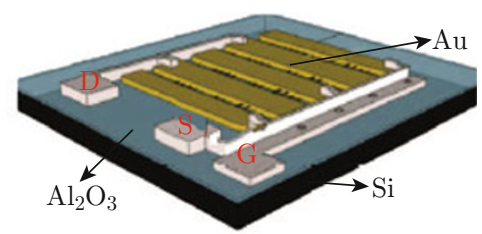

(b)

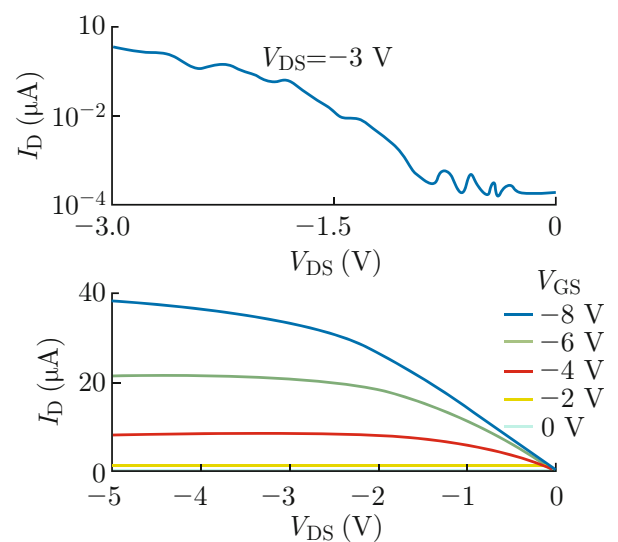

(c)

Fig. 4 (a) CNTs transferred on the silicon substrate with already patterned gates arrays; (b) FET built on the top of the transferred nanotubes; (c) p-MOS like ID-VG and ID-VD characteristics of the CNT FETs.

they use photolithography technique to get the contact holes and wires in order to build the circuits. Since the CNT transistors they used are only p-type based FETs, a series of p-channel MOS (pMOS)-only logic units are adopted to build the whole computer system. The mini-computing system is composed of $178 \mathrm{CNT}$ FETs, with each FET comprising about 10 to 200 nanotubes, depending on the current drive of each FET. The system is fabricated using the university lab with just $1 \mu \mathrm{m}$ lithography and limited measurement capability. Even though the fabrication is not optimized and not the state-of-the-art, they have achieved $1 \mathrm{kHz}$ operating computing unit to execute simple million instructions per second (MIPS) instructions. Stanford's group demonstrates the first time the feasibility of us- ing CNTFETs and the related logic circuits to build an operating computer unit. With the more-advanced facilities in the modern Integrated Circuit (IC) industry it is very promising to implement CNTs in a more effective way to build a more optimized computing system.

\section{Nanosensor}

A sensor is a converter that measures a physical quantity and converts it into a signal which can be read. Nanosensors means the sensing elements are made of nanomaterials, including nanoscale biological and chemical sensors, nanosensors on flow, pressure and temperature, etc. Development of nanotechnology not only provides high sensitivity for sensors, such as 
nanoparticles, nanotubes, nanowires and films, etc., but also provides many novel ideas and techniques to make sensors towards nanometer scales. Compared with traditional sensors, nanosensors have smaller sizes, improved accuracy and performance, etc. More importantly, nanosensors function on the atomic scale, which greatly enrich the theory of the sensing and broaden sensor applications.

Major application areas of nanosensors include health care, military use, industrial control and robotics, networking and communications, and environmental monitoring. With nanotechnology maturity, nanosensors show a great advantage in the defense and securitychecking areas. In the near future, nanosensor uniform and equipment will be used to detect the risk of anthrax and other gases.

Currently, MOS and Single-Electron (SE) sensors are major gas sensors in the market [43-45]. But both are working at higher temperatures, and they have high power consumption, low sensitivity, poor antiinterference ability, and they are difficult to use [4648]. With the development of nanotechnology in recent years, there are extensive publications in nano gas sensors. Especially, carbon nanotube gas sensors have a significant progress [49-51]. CNT resistive sensor (Fig. 5(a)) and FET sensor [52] (Fig. 5(b)) are widely used to detect the changes of the ambient gas molecules [53]. Because the conduction of CNT is almost entirely dominated by the atoms from the surface, even a tiny chemical change in surrounding environment gas will cause a considerable conductivity change. For example, a semiconductor-type CNTs in air environment is a p-type $[54,55]$. When in a reducing gas, if the charge transfer occurs, electrons enter the CNTs to recombine a hole, which results the conductance in CNTs decrease, making the CNTFET $I-V$ curve move to a negative $V_{\mathrm{g}}$ direction. Similarly, if the oxidizing gas is in contact with CNT, electrons of the CNT will be stolen by the surrounding gas, conductivity increased, CNTFET $I$ - $V$ curve then moves to the positive voltage direction of $V_{\mathrm{g}}$.

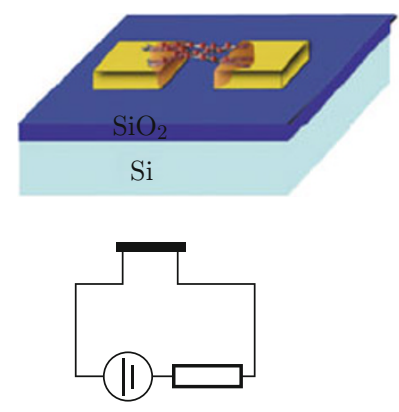

(a)

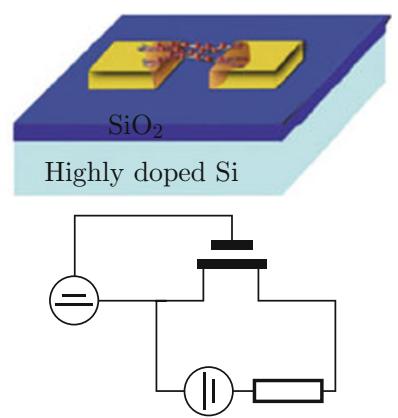

(b)
Fig. 5 The schematic of (a) A chemical resistive sensor; (b) A chemical field effect transistor sensor.

From 2000 Dai research group [56] first reported CNT based environmental gas sensors. Till the end of 2009, there were more than $4100 \mathrm{CNT}$ sensors publications in scientific literature [57-61]. Figure 6 shows detailed statistics published annually by the CNT sensor scientific literature. From the figure we can see that the literature on this subject continues to increase each year, indicating that CNT sensor is still hot research topic. Our following review is an overview of CNT gas sensors for environmental monitoring, medical testing and other national defense and military use. Carbon nanotube commercialized gas sensors are also compared and forecasted.

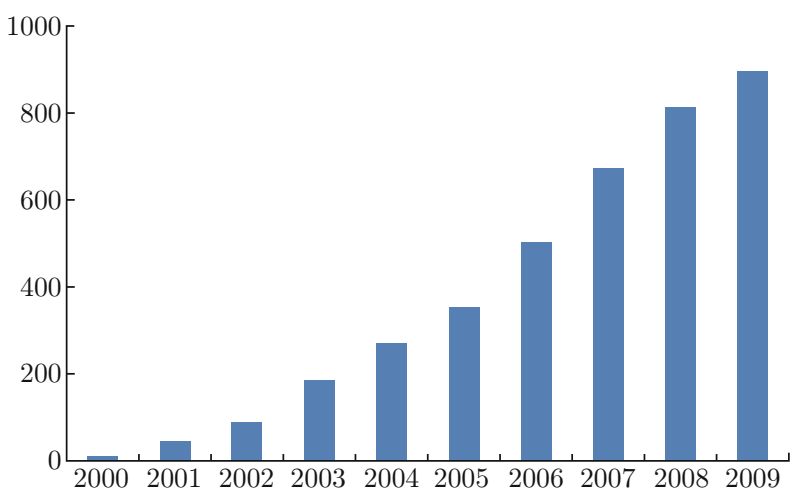

Fig. 6 Published articles on Carbon Nanotube sensors from Year 2000 to 2009. (Statistical source: ISI Web of Knowledge)

The complexity of the modern battlefield and increased terrorism make it particularly necessary in dangerous gas detection. 2,4,6-trinitrotoluene (TNT), cyclotrimethylenetrinitramine nitramine (RDX), and ethylene glycol dinitrate (EGDN) are often detected as high explosives. Chemical warfare agents (Chemical War Agent, CWA), particularly nerve gas, often highly toxic, are extremely dangerous gases. Nerve gas typically reduces the activity of many enzymes, resulting in human body neurotransmitter - acetylcholine highly aggregated, and poisoning human nerve. This activity is very rapid and irreversible, causing people to die in a short time. Current reported sensors including nerve gas sensors, electrochemical sensors, chemical resistance sensors (such as MOS sensor, SAW sensors), etc. [62], are coated with a layer of chemistry resistive material on the surface. To detect nerve gas, a stimulant gas is usually used instead of actual poisonous gas. Such as to use Dimethylmethylphosphonate (DMMP) to replace sarin gas, methyl phosphonate (Diisopropyl Methanephosphonate, DIMP) to replace soman gas, thionyl chloride $\left(\mathrm{SOCl}_{2}\right)$ to replace nerve gas in the initial stage of experiments, to prevent accidents and reduce the experimental risks.

Our research group reported functionalized SWNTs using APS, and a self-assembly method in $\mathrm{Si} / \mathrm{SiO}_{2}$ surface to make SWNT sensor network. The sensor showed 
good response on 5 ppm of DMMP vapor at room temperature. The sensor response was rapid, reversible and repeatable. The density of SWNTs in the sensors could be controlled by suspension concentration and the deposition current. It was found that a density of 30-40 tubes $\mu \mathrm{m}-2$ showed the best performance of detecting DMMP. Figure 7 shows the sensor testing system and the resistance response curve.

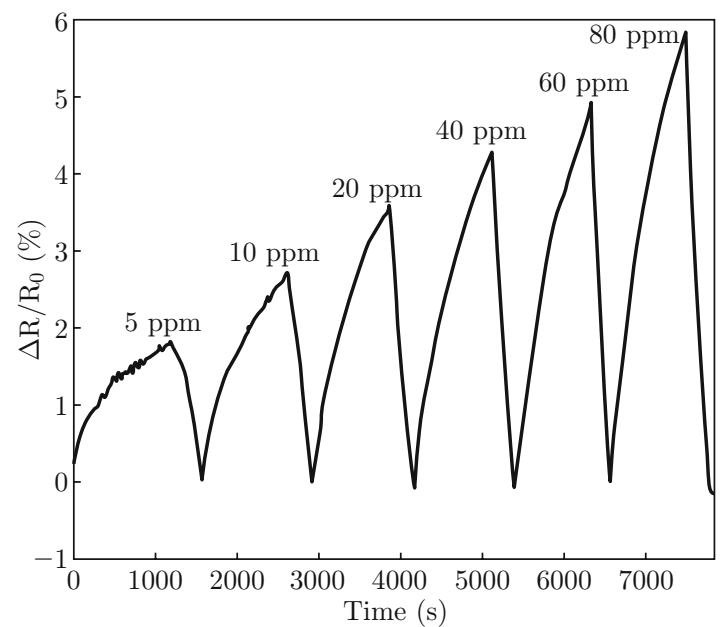

Fig. 7 CNT gas sensor response to different concentrations of the gas resistance.

CNTs' huge aspect ratio and surface area make them highly sensitive to adsorbed material layer that could efficiently change the conductance of the CNT channel. CNT gas sensors can be used in various industry, environmental protection, safety, medical testing, and defense and military situations. The current study focuses on increasing selectivity of the sensors to a practical detectable level.

CNT gas sensor technology [63] is currently in the early stage of development. For practical applications, there is still a long way to go. Improving the CNT gas sensor sensitivity, selectivity, and interference immunity are currently the most important issues. Conventional sensors have been very well developed, and yet introducing the CNTs into these sensors can greatly increase their sensitivity. Certain metals, metal oxides and polymers will generate electrical response with a specific gas molecule. If CNT is used to identify substance the selectivity of the sensors can greatly be enhanced. Meanwhile SAW and ionization sensors, etc. are also proven to improve the sensor selectivity. CNT gas sensor selectivity improvement still needs further in-depth study. The interference immunity can be achieved by either reducing interference or offsetting the interference. Using the non-interactive interference coating source on the CNT can reduce interference. Finding materials which have opposite response to the disturbance on CNT would counteract the effect of interference. In the course of solving the above technical problems, CNT gas sensors also need to consider the reality of their social and environmental impact. CNTs toxicity to organisms and harmfulness to the environment need further detailed assessment. Desirable sensor development and process are environmentally friendly and should be compatible with the existing technology, which greatly reduces the CNT gas sensor commercial costs.

It is seen that a small, stable, durable, portable and low-power sensor will be available in future. CNTs can be easily integrated into micro-electronic devices and make the sensor array by many mini-sensors. With the development of microelectronics technology, manufacturing costs continue to decline. Similar to other electronic products, CNT sensor production will also be more affordable. Cheap disposable sensors with CNTs can greatly help medical diagnosis and personal protective applications. Conventional sensors require high temperatures to operate, which increases the power consumption and shortens lifetime of the sensing element. CNT sensor can operate at room temperature, with smaller size and lower power consumption, so that the core device has longer lifetime. Combining CNT sensor technology together with other advanced technologies, such as Micro-Electro Mechanical Systems (MEMS) [64], wireless communication technology and IC design, etc., will likely bring a revolutionary change in sensing applications.

\section{Photovoltaic nanodevices}

Nanomaterials add new vitality to solar cell development. Feature size of nanomaterials is from 1 to $100 \mathrm{~nm}$. In this range, many new effects such as surface effect, small size effect, quantum-scale effect, and macroscopic quantum tunneling effect, etc. could be significant and observed. These, in turn, yield "bizarre" mechanical, electrical, magnetic, optical, thermal and chemical properties. Solar cells based on nanomaterials are considered as one of the next generation of high-performance and low-cost solar cell options.

In recent years, there are already some developments in specific optical properties in nanomaterials, nanotechnology and composite functional structures/devices. There are plenty of nanomaterials with special optical properties which can be used, and there are various assembly methods which can be used to build photovoltaic nanostructure. Assembly consumes fewer materials, the manufacture processes are more controllable, and can be done under non-vacuum environment in a large scale. Study of composite functional assembly of photovoltaic devices includes the scale, level, interface, texture, and controllability, optoelectronic functional recombination, improving the photoelectric conversion efficiency, improving the stability of amorphous silicon thin film photovoltaic cells and making use of a full spectrum of light in the new gener- 
ation photovoltaic cells. These research activities have very important practical and strategic significance on our national economic, social and technological development.

A semiconducting SWNT is of specific optical properties: direct band gap, symmetric conduction and valence bands, energy level separation structure, good light response from ultraviolet to infrared spectrum, multiple exciton generation (MEG effect) when absorbing photons, multi-photon absorption (known as MPA effect), and infrared photons absorptions (more electrons generated through the two photon-photon excitation to higher energy excited state) performance. Therefore, semiconducting SWNTs are almost ideal material for photovoltaic cells, which can make use of the incident photons that the ordinary photovoltaic cells cannot use. In addition, in July 2007, the national renewable energy laboratory (NREL) in U.S. also found the silicon nanoparticles can effectively generate MEG effect, improving the crystalline silicon photovoltaic cell conversion efficiency from $30 \%$ to $44 \%$. To develop and utilize MEG and MPA effects greatly improves photovoltaic conversion efficiency on conventional photovoltaic cells by converting the high-energy photon energy into electrical energy (which is generally wasted as heat loss in ordinary photovoltaic cells).

Interaction between the incident light and free charges on the metallic nanoscale surface yields surface plasma, and the light field can be conducted along the metallic nanowires and be intensified locally. This causes localized-enhanced light absorption and photoelectric conversion. Nanometal wires attached onto photovoltaic cells generate surface light scattering and propagation. When properly assembled, this allows more light to enter photovoltaic cells, to be captured and absorbed more effectively. Furthermore, approximately $12 \%$ of the solar spectrum is above $3 \mathrm{eV}$ and this portion of light can only penetrate into the surface layer less than $200 \mathrm{~nm}$. In crystalline silicon solar cells, the n-type layer window has to be very shallow (generally $<100 \mathrm{~nm}$ ) in order to improve the utilization of high-energy photons, giving rise to a high series resistance of n-type surface layer, reducing the conductivity and collection of photoelectric current. Adding metal nanowires in the n-type surface layer could solve the problem of high series resistance. Furthermore, $\mathrm{SnO}_{2}$, etc. [65] oxide nanowires with wide band gaps have high transparency and conductivity. They could form waveguide light transmission and modulation; the features that can also be used to transfer current as well as incident light on photovoltaic cells surface.

Crystalline silicon photovoltaic cells have a large area of metal electrode on the surface window, which blocks about $2-5 \%$ of the total incident sunlight, resulting in proposing a complete-back-electrode crystalline silicon photovoltaic cell. However, due to the alterna- tive arrangement of positive and negative electrodes on the back, PN junction is on the backside, causing a low conversion efficiency of this kind of solar cells. For concentration-type of photovoltaic cells, metal electrode may block more incident lights, about 6-10\%. To resolve this issue, we then proposed: (1) to introduce $\mathrm{Ni}$ nanowires in the surface of the metal electrode window, forming a surface waveguide plasma channel. The incident light could interact with the surface free charges of Ni nanostructure, forming the surface plasma waves, propagating light along $\mathrm{Ni}$ nanowires on the surface into the edge area of the electrode and then achieving the photoelectric conversion. This led to localized enhanced light absorption and strong injection efficiency for photoelectric conversion; (2) $\mathrm{SnO}_{2}$ nanowires has a smooth surface, uniform diameter, and wide optical band gap. It can be used as a nanoscale waveguide. Using $\mathrm{SnO}_{2}$ nanowires on the surface to build a waveguide structure can make the incident light efficiently coupled into the nanostructure in the light guiding cable, transmit incident light in the guided mode. Transmitting incident sunlight into blocked area improves the efficiency of photovoltaic cells. Conventional crystalline silicon solar cells have front metal gate line area of about $8 \%$ of total area, of which $60 \%$ is a thin gate line (finger, also known as sub-gate line), $40 \%$ the main gate line (bus bar). Metal gate are usually composed silver particles and opaque vitreous. Reducing gate lines blocking on incident light can greatly reduce the loss of cell efficiency.

The phenomenon that metallic nanostructures generate surface plasma and enhance photovoltaic conversion is becoming a hot research topic. Schaatd etc. deposited 50, 80 and $100 \mathrm{~nm}$ gold particles on p-type silicon and enhanced photocurrent by $50 \%-80 \%$ at 500 $\mathrm{nm}$ wavelength. Derkacs etc. used $100 \mathrm{~nm}$ gold particles in amorphous silicon solar cells and increased the short-circuit current by $8.1 \%$ and energy conversion efficiency by $8.3 \%$. Pillai et al. enhanced the light absorption by 16 times through depositing silver nanoparticles onto the surface of silicon-based thin-film solar cell substrates. Atwater's team obtained 2.5 times of the absorption enhancement at $1100 \mathrm{~nm}$ wavelength of light by simulation on surface plasma excitation grating structure on the back of the silicon thin film of the solar cells. As can be seen, using excited surface plasma by metallic nanostructures to enhance the absorption of photovoltaic cells and, hence, to improve the energy conversion efficiency is a feasible approach. Surface plasma on nano metal particle surface can greatly enhance light field oscillations within the particularly metal nanowires, which is a plasma waveguide effect. This is equivalent to a nanoscale antenna and has a great potential for composite photovoltaic cell development. We have used a magnetic induction method, coupled with the magnetic field in the solution with- 
out a template, to reduce nickel ions (or thermal decomposition of organic nickel compounds) through the dipole-dipole interaction along the direction the magnetic field lines and grow into $\mathrm{Ni}$ nanowires. Then, the Ni nanowires were assembled to the n-type shallow junction on the surface window of crystalline silicon photovoltaic cells, forming a compound photoelectric converter. Significant improvements in the performance and efficiency $(>5 \%)$ have been achieved. Transparent conductive oxide nanowires have wide band gaps. They also have high conductance and modulate the light through the nanowires. They can be used as photovoltaic cell incident surface electrode to conduct current. Through controlled growth of $\mathrm{SnO}_{2}$ nanowires by high-frequency induction heating evaporation method, we successfully grew $\mathrm{SnO}_{2}$ wires using $\mathrm{Sn}$ as the base material and appropriately controlling the oxygen content in the carrier gas.

The key for composite nanostructure of photovoltaic cells is to assemble different nanomaterials, different structures, scales, shapes, densities, distributions, spacing, materials, etc. in a cooperative way to achieve the full spectrum solar photoelectric conversion. In addition, studies on the impact of photo-generated electrons by nanomaterial hetero-junction, interface modification and surface modification, inhibition of adverse effects, and enhance synergy mechanisms are extensively ongoing. Optoelectronic devices probably need to incorporate diverse optical nanomaterials. Flexibility and the controllability are important directions on developing low-cost/high-performance photovoltaic cells. Then we now controllably fabricate a system of different optoelectronic devices with morphologies, characteristics, photoelectric conversion mechanisms, optoelectronic devices machined components and isomers morphology of specific device. Isomers may be flat or threedimensional shape, chiral or oriented, crystal or amorphous. There are many types of controlled assembly methods: self-assembly method within micro-solution, the fluid surface method, surface micro-infusion solution evaporation method, a spray coating method, and printing/embossing method. Printing technology can also be used in solar industry. Cornell University (New York, Ithaca) and DuPont, (a materials science and engineering company in Delaware, Wilmington) $[66,67]$ invented simple chemical processes using semiconductor "ink" to print a photovoltaic cell. The inks can be directly printed as a thin layer of flexible photovoltaic (PV) materials.

As a summary of the above research work, to assemble nanocomposite into solar cells is a new concept and a viable idea. This makes the solar cell more costeffective by a fully-utilized spectrum of solar light. (1) High energy photon greater than the semiconductor energy gap can be effectively converted into electricity with composite assembled carbon nanotubes; (2) En- ergy of the infrared photon less than the semiconductor band gap can be used by introducing up-conversion fluorescence nanomaterials into composite structure to achieve effective photoelectric conversion; (3) Nanowire waveguide can transmit light to the electrode edge of photoelectric conversion area for effective collection and utilization, a solution to solve the problem of the blocking incident light by surface electrode; (4) Introducing carbon nanotubes, down-conversion fluorescent nanomaterials or UV fluorescence nanomaterials into solar cells improves not only the incident light photoelectric conversion, but also the stability of amorphous silicon thin film solar cells.

Solar photovoltaic system is mainly used to convert solar energy into electrical energy and then chemical energy and store it in battery. To charge the battery during daytime we usually make use of the left-over solar electrical energy of the day, depending on loadings. The battery is therefore in a semi-floating charge state. Chemical energy storage in a solar photovoltaic power generation system is a major application bottleneck and has the following challenges: (1) The energy density of battery storage system is relatively low, while the cost is high; (2) The charge rate is low and the performance degrades with time. However, the floating charge voltage is low, float current is small, easy to maintain, has no corrosion, no pollution, no gas, no liquid spill, and environmentally friendly, etc. Currently nanotubes, nanorods, nanowires and other nanomaterials can be used as the positive and negative electrodes in lithium-ion batteries. There have been extensive publications available in this research area.

\section{Multi-exciton effect photovoltaic cells}

Multiple exciton generation (MEG) effect refers to the generation of multiple electron-hole pairs from the absorption of a single photon. It was discovered as early as 1950s in the semiconductor bulk materials. Till recently, it is noted that MEG may considerably increase the energy conversion efficiency of nanocrystal based solar cells.

In 2004, Schaller and Klimov reported a strong multiexciton effect in semiconductor nanostructures. This triggered a wide and deep study [68] on multiple exciton effect in semiconductor quantum dot structure. So far, significant MEG was observed in PbSe, $\mathrm{PbS}$ [69], $\mathrm{PbTe}$ [70], CdSe [71], Si [72] and InAs [73], and other semiconductor nanostructures, respectively. It was found that in the 0.5 to $3.5 \mathrm{eV}$ surface solar spectral regions, silicon nanoscale crystals have very strong multi-exciton effect. The photon threshold was 2.4 times of the band gap and at 3.4 times band gap position, the quantum yield reached $260 \%$, far better than crystalline silicon (Fig. 8). Till recently, multiple exciton generation effect, virtual exciton generation theory, coherent exciton 

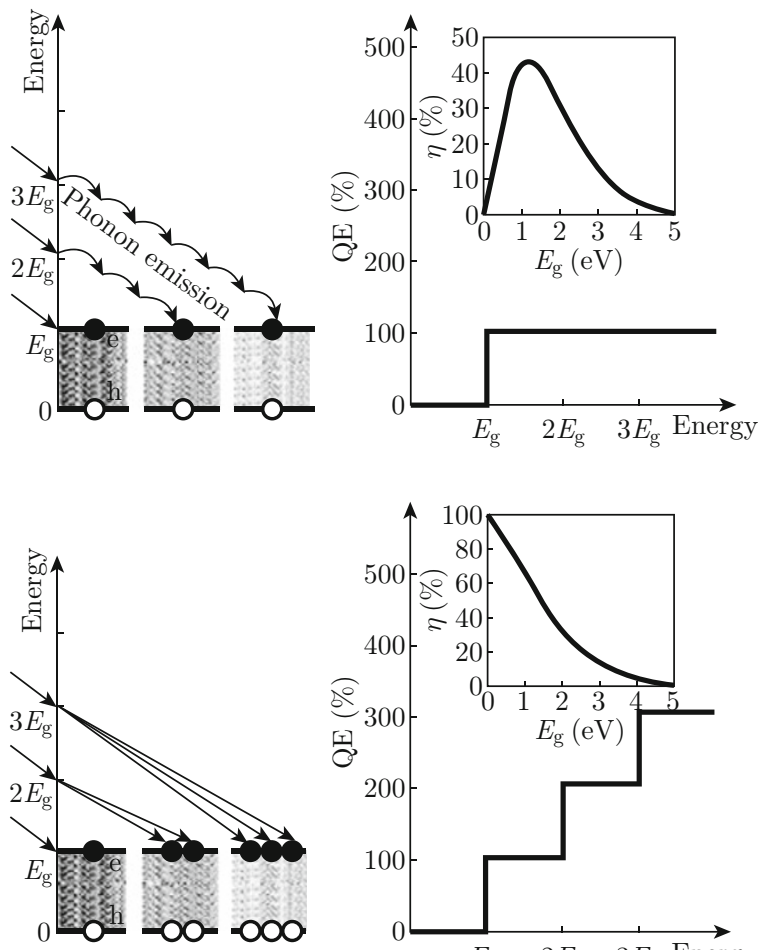

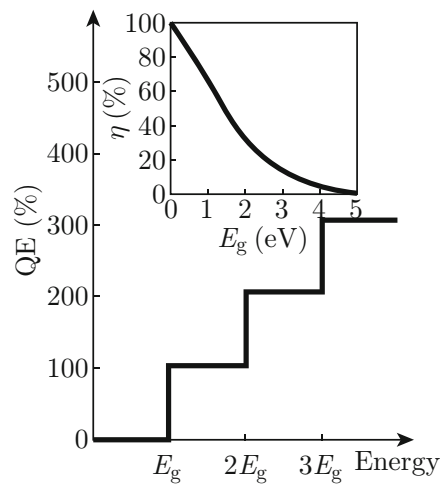

(a)

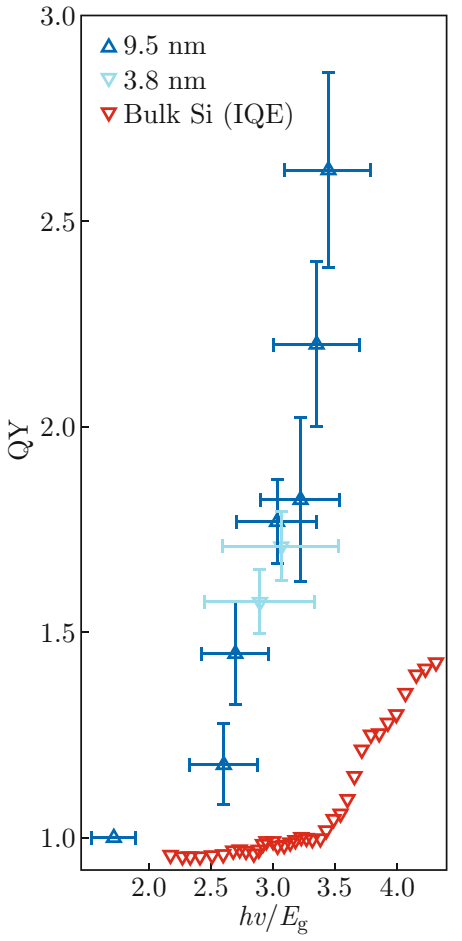

(b)

Fig. 8 (a) Schematic of a single and multiple electron-hole pair (s) generation process by a photon; (b) Multiple exciton effect in silicon nanostructures and in bulk silicon.
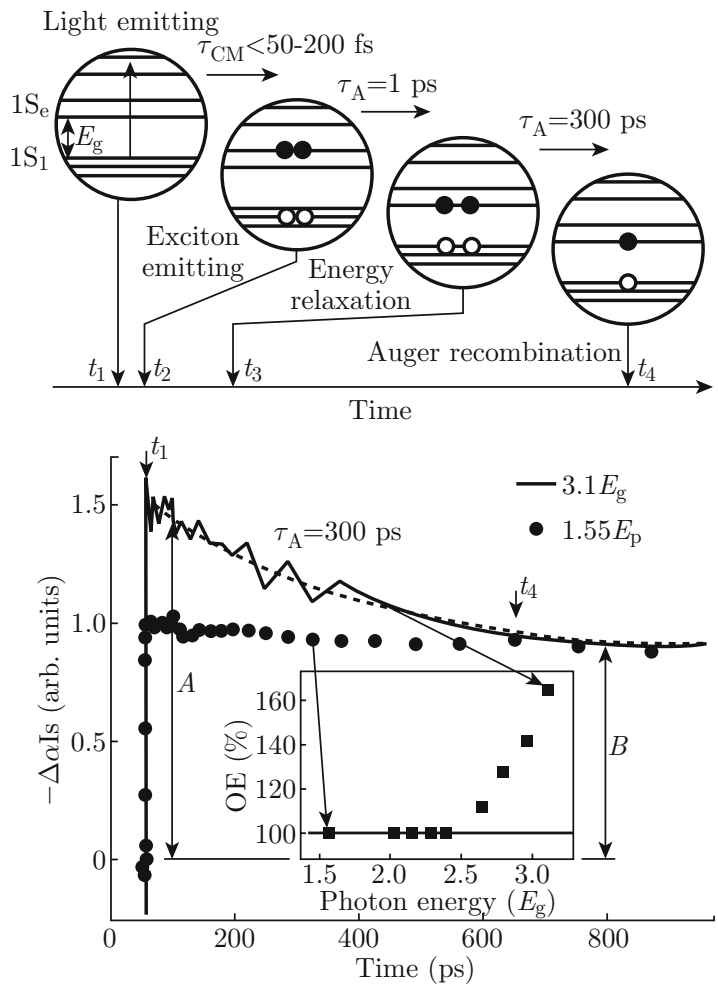

(a)

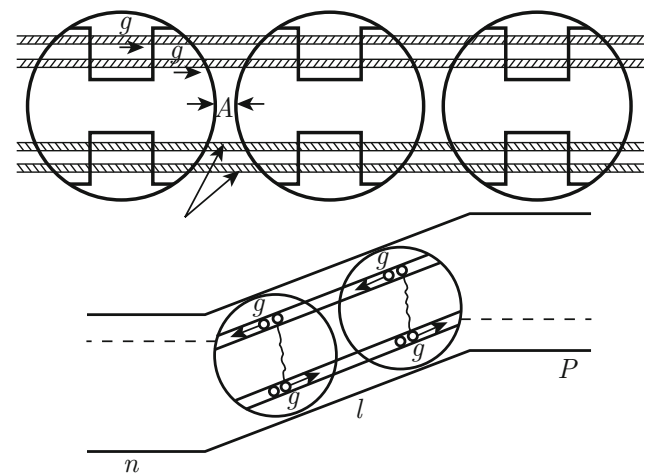

(b)
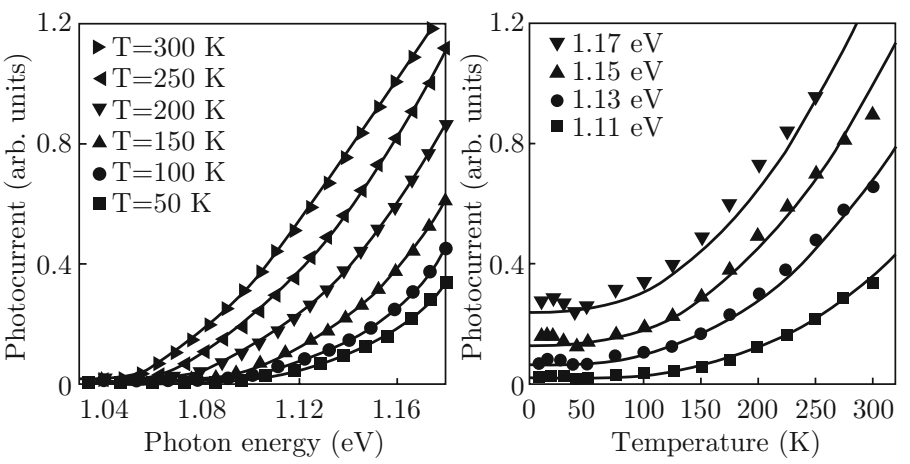

(c)

Fig. 9 (a) Observation of multiple exciton effect by pump-probe experiment; (b) Miniband from electron wave functions coupling in quantum dot array p-i-n solar cell; (c) Miniband observed experimentally in nanocrystalline silicon thin film. 
model and multi-impact ionization mechanism were proposed to interpret the MEG effects.

Generally, when a semiconductor material absorbs one photon, one single exciton is generated. The energy of high excitation energy photon is wasted by phonon scattering. If multi-excitons by one photon can be achieved (Fig. 9(a)), the conversion efficiency of high energy photons can be significantly improved. Actually, it has been confirmed by many experimental observations that MEG in nanomaterials can improve photoelectric conversion efficiency of solar cells [74]. Theoretical calculations showed that in PbSe nanocrystals, this new cell conversion efficiency would be expected to increase by $50 \%$. Therefore, design of multi-layer film battery with MEG effect is the latest research hot topic. The main purpose of this new research is to make full use of the hot carriers from thermal relaxation to the semiconductor band edge so as to significantly improve cell conversion efficiency. This could achieve double (or more) electron-hole pairs by impact ionization then increase in the photocurrent, requiring impact ionization rate higher than the hot carrier cooling and other relaxation processes.

At this stage, study of MEG effects in nanostructured silicon solar cells has just begun. There are still many technical issues to be resolved to achieve this multiexcitonic hot carrier effects in solar cells. The biggest difficulty is the effective charge separation: we have to control the time longer than multiple exciton generation time $\left(10^{-13} \sim 10^{-12} \mathrm{~s}\right)$, but less than the dualexciton lifetime $\left(10^{-10} \mathrm{~s}\right)$. There is no doubt that this semiconductor-based MEG effect in the new hot carrier solar cells with silicon nanostructures, once achieved, is bound to have a revolutionary impact on existing photovoltaic science and technology. It is known that in the quantum dot p-i-n solar cell array, quantum dots coupling between the electron wave function formed by minibands (Fig. 9(b)) slow down the hot carrier cooling rate, making the hot carriers transmitted and collected in the respective $\mathrm{p}$ and $\mathrm{n}$ electrodes [75]. In the uniform nanocrystalline silicon thin films, since the amorphous grain silicon is very thin (only 2 to 4 atomic layers), the electron wave functions in silicon quantum dots very easily couple each other.

Solar Energy Research Institute of Shanghai Jiaotong University has observed the band tail states transitions in amorphous $\mathrm{Si}$ and confirmed the existence of miniband coupling in nanocrystalline silicon thin films [76] (Fig. 9(c)). This brings hope on exploring hot-carrierbased nanocrystalline silicon thin film battery cell. Specific technical directions includes the use of optimized semiconductor nanocrystals; characterization of the frequency response of the device under illumination of different frequencies throughout photocurrent spectra; exploration of MEG effects on photocurrent, voltage, and energy conversion efficiency; optimization of the sepa- ration and collection of multiple electron-hole pairs in nanocrystalline silicon thin-film cells to improve solar cell conversion efficiency, etc.

Single-wall carbon nanotube is of many specific optical properties: carriers can one-dimensionally ballistic transported, no degradation on carrier mobility, direct band gap, symmetric conduction band and the valence band, energy level with separation structure, good response from the ultraviolet to the infrared region of the spectrum. Therefore, semiconducting single-walled carbon nanotubes are nearly ideal photovoltaic cell material and can efficiently absorb incident photons (useless for typical photovoltaic cells) to produce more electrons.

In 2008, we developed a single CNT based micro-solar cell [77]. As shown in Fig. 10, an array of semiconductor SWNTs were fixed to the end of metal electrode with different work functions. SWNT internally generates electric field for the separation and collection on photo-generated electron-hole pairs. Under sunlight, this micro-cell can achieve an energy conversion efficiency of $12.6 \%$.

In 2009, researchers at Cornell University replaced the traditional silicon with carbon nanotubes and manufactured a highly-efficient solar cell [78]. Their device was a CNT based photodiode (Fig. 11). It was a simple solar cell, converting light efficiently into electricity with multiplied current. CNTs were connected between two electrical contact points which were close to the negative and positive gates. By using different wavelengths of laser irradiating on different sizes of nanotubes, high current multiplication was observed. Further studies revealed that the narrow cylindrical structure of CNT can pile one electron onto another. Electron through the nanotube is excited during migration and then generates electric current.

\section{Multi-photon absorption}

Multi-photon absorption is a nonlinear optical effect. With a high-intensity laser beam irradiation, a material may absorb several, or even dozens of photons; this effect is called multi-photon absorption. This phenomenon can be interpreted as multiple photons being absorbed simultaneously from initial states to the final states, via a few dummy intermediate states.

Multi-photon absorption process may be accompanied by multi-photon emission, electron conduction, optical-electric interaction, fluorescence, dissociation, photochemical reactions, and other multi-photon effects. These phenomena in turn help to study the multiphoton absorption process. Multi-photon absorption process is a high-order nonlinear optical process and it is closely related to the material damage threshold, which is important for theoretical solid state physics studies (for example, to understand details on the band 


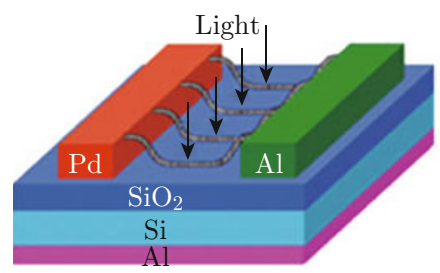

(a)
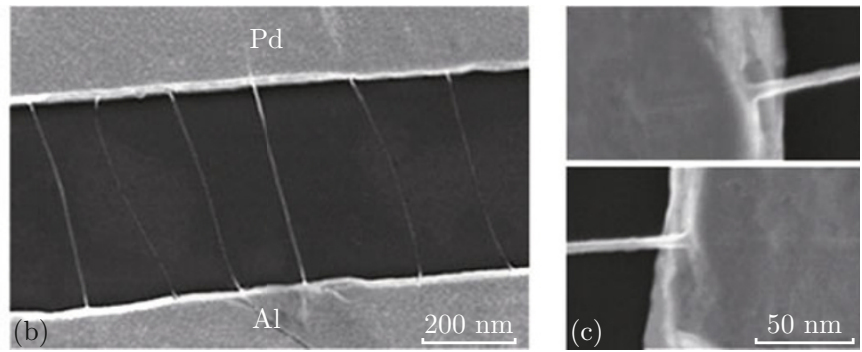

Fig. 10 SWCNT-based micro photo cell (a) Schematic diagram; (b, c) SEM Photos.

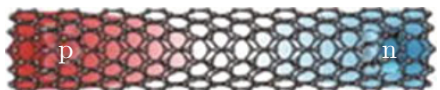

SWCNT PN junction

(a)

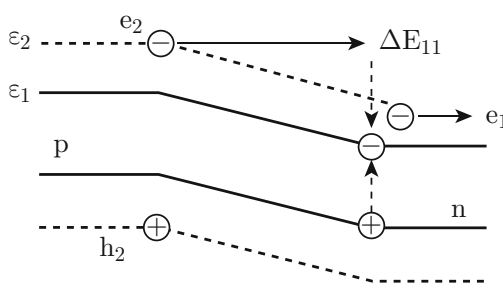

Multi electron-hole-pair generation

(b)

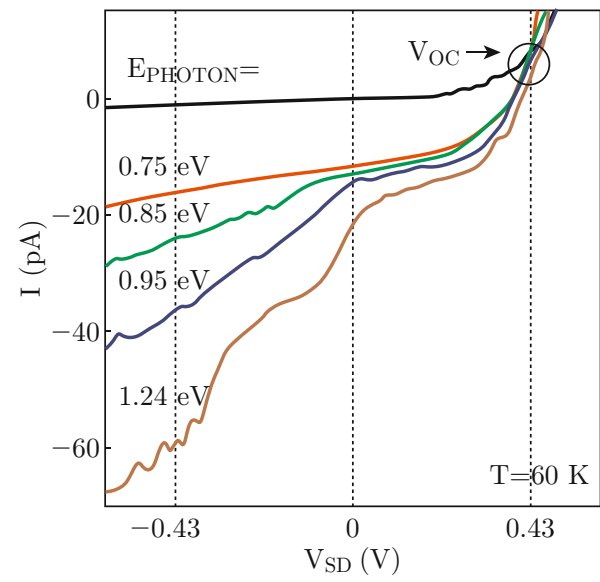

Photo current vs. Voltage (different photon energies)

(c)

Fig. 11 Single-walled CNT PN junction.

structure and the interaction between ultra-short light pulses and semiconductor). Laser-irradiation-induced multi-photon absorption process has been successfully used for the separation of isotopes of sulfur, and has important applications in spectroscopy, materials research, and photochemical isotope separation areas. Because of its importance in nonlinear absorption of high-power laser technology and basic research in solid state physics theory, especially exploring the energy band structure information, multi-photon absorption process is receiving more and more attention.

Two-photon absorption happens on one same atom or molecule which could simultaneously absorb two photons (same or different energy) in one process. Since laser intensity is proportional to the square of twophoton absorption, the absorption process can produce limited-space excitation, which has a great potential application in two-photon confocal lasers, scanning microscopy on three-dimensional imaging, two photon pumped up conversion lasing, two-photon optical limiting, three-dimensional optical data storage, photodynamic therapy and two-photon-induced biological cage baskets research, etc. Efficient two-photon sensitive material development has brought many technical applications, and this also draws research interests in three-photon excitation. Three-photon absorption in- volves three photons induced electron transitions to the high energy levels. As three-photon absorption is for longer wavelength photons, penetration of the photons into the absorbent material is improved and deeper observation can be achieved. In addition, the threephoton process is directly proportional to the cube of incident light intensity; this brings much higher spatial modulation and higher image contrast.

A three-photon absorption phenomenon was first observed in 1964. Since then, materials with cross section of three-photon absorption were frequently reported, including liquids (like solvents), solid (like semiconductors and ceramics) as well as gases. Since threephoton absorption relates to the fifth-order nonlinear process and is difficult to be observed experimentally, little has been reported about this phenomenon. Until the last decade, the high repetition frequency tunable solid-state femtosecond (FS) laser provided the most stable high-energy pulsed excitation source for multiphoton-absorption-induced fluorescence. This enables the research and study on multi-photon absorption application.

In the past ten years, theoretical and experimental studies are mainly focused on two-photon absorption relationships between the material structure and properties. The results showed that two-photon absorption 
cross section could be affected by molecular structure of effective conjugation length. The longer effective conjugation length results the larger two-photon absorption. Molecular structures with A-D-A or D-A-D were found to have larger two-photon absorption. The higher the concentration of co-planar molecules results the greater the two-photon absorption. In addition, the polarity of the solution also has influence on two-photon absorption.

In short, increasing the molecular two-photon absorption is the key to enhance intra-molecular electron delocalization, which increases the degree of charge transfer. For example, we can increase the length of the bridge of conjugated bond (this actually increases the charge transfer distance), or change the terminal electron donor or electron acceptor force, and introduce electron withdrawing cluster on the side chain of the central bridge conjugate bond. In addition, other researchers are studying the quadrupole and octupole moments, multi-branched and dendritic molecular structure. The results suggest that increasing the dimension of intra-molecular charge transfer would also increase the two-photon absorption cross section [79].

Multi-photon absorption can be used as the upper or lower layers of a solar cell. This will narrow frequency band of the incident light, more adaptable to the energy band width of conventional PN junction solar cell, so as to improve light absorption and increase the efficiency of solar cells [80].

\section{Light trapping structure of solar photovoltaic cells}

The light losses in solar cells are from the three main areas: (a) Surface reflection losses; (b) Energy loss of the incident light due to surface electrode material coverage; (c) Transmission loss due to the thin films in the cells.

We introduce optical light trapping structure to deal with the light transmission loss: (a) Coat or deposit anti-reflective surface texture onto light-receiving surface; (b) Lengthen the light absorption layer. For example, increase the refractive index of the absorption layer in between the upper and lower textured materials. Back reflection is enhanced so that some light is returned back to the cell as the second absorbent; (c) Minimize the area covered by the gate electrode on the surface to maximize the output photocurrent. Low absorption rate of long-wavelength photons would decrease light current density when material thinning down to $50 \mu \mathrm{m}$. This loss can be compensated by introducing a light trapping structure, increasing the collection efficiency with the aid of theoretical calculation of the optical path [81].

Current commercial crystalline silicon solar cells have a typical thickness of more than $5 \mu \mathrm{m}$. Light trapping is then achieved by coating an anti-reflection film or using suede on the surface. Suede has a better effect than anti-reflection film. Therefore, crystalline silicon solar cell with suede technology is good measure to improve cell efficiency. (a) Alkaline etching method is often used to build a surface pyramid-like structure for crystalline silicon solar cells; (b) For poly-silicon cell, surface anti-reflection is generally used where the coating may depend on the silicon material. The efficiency of polycrystalline silicon cell has reached $16.8 \%$ in laboratory. Thus, the poly-silicon suede technologies are not yet widely used in large-scale production. The key is how to build a uniform, stable suede microstructure on polycrystalline silicon and to make a good interfacial contact between them.

Using an anti-reflection surface alone is not sufficient to trap light to build silicon-based thin film solar cells. Transparent electrode and back-surface reflection are also used in the light trapping structure. Currently suede $\mathrm{SnO}_{2}$ transparent conductive glass has been industrialized. But using $\mathrm{SnO}_{2}$ as a substrate and coating a plasma enhanced chemical vapor deposition (PECVD) $\mu \mathrm{c}$ : Si:H films on top of it, the hydrogen plasma could make $\mathrm{SnO}_{2}$ unstable and reduce it into in black color. The $\mathrm{ZnO}$ film in hydrogen plasma has better stability and doping $\mathrm{ZnO}$ thin films achieves a comparable performance with the indium tin oxide (ITO) and $\mathrm{SnO}_{2}$ film. Therefore, in the $\mu \mathrm{c}$ : $\mathrm{Si}: \mathrm{H}$ thin film solar cells, the use of $\mathrm{ZnO}$ :Al film as the transparent electrode material is a future trend.

With reducing solar cell thickness, the cell performance is limited in two aspects. First, the transmitted light increases as the thickness decreases. The indirect band gap materials have more losses than direct band gap materials. Second, back electrode influence becomes significant. Theoretical calculations by Pelanchocf on $50 \mu \mathrm{m}$ c-Si n+-p solar cells showed that when the cell material reduces to $50 \mu \mathrm{m}$, the recombination of light induced carriers is also reduced. Therefore, the cell thinning reduces absorption rate of long-wavelength photons then reduces the photocurrent density. Light trapping structure based on optimizing the optical path may improve the collection efficiency and compensate this thinning effect. As thickness of the cells is reducing, the light trapping effect is becoming more and more necessary $[82,83]$.

Light trapping structures can be implemented through: (a) Reducing the input light reflection by depositing anti-reflective film or other surface texture; (b) Increasing light absorption path in the cells. Making the refractive index of the absorbing layer greater than the upper and lower materials and strengthening textured back reflection; (c) Reducing the gate electrode coverage and maximizing the output photocurrent. 


\section{Conclusions and future prospects}

In recent years, many achievements in nanoelectronic devices and circuit theories have been made. Advances in nanomaterials and nanoscale fabrication technologies have made many conceptual nanodevices into prototypes. Although nanoelectronic device technologies have great advantages, there still exist many challenges on each stage. Most devices are still in the laboratory research stage. Some nanodevices do not have desirable reliabilities and repeatability. Some only work under certain conditions and they may not maintain their performance under other environments. In short, more research efforts are required for most nanodevices to be released to the markets.

From technology development perspective, there are a few urgent issues needs to be resolved:

(1) Fabrication of quantum nanoscale heterostructures

To miniaturize electronic devices down to the nanoscales, the most straight forward approach is to use epitaxy, lithography and other sophisticated technologies to fabricate a sandwich-like nano semiconductor structure where the different layers are different semiconductor materials with different potentials to form a series of quantum wells.

(2) Molecular transistors and wires assembly of electronic nanodevices

Even if one knows how to make nano transistors from molecular wires or nanotubes, to assemble the nanostructures into desirable patterns according to design principles is still a challenging job.

(3) Ultra-high-density memory cell using quantum effects

Memory chips to store ultra-high-density data will be a key to build future nanoscale computers. This chip can provide fast data access and huge storage room. To build this high density quantum memory array by making use of the existing logic nanodevices is a new challenge.

(4) Nanoscale "interconnection"

Assembling trillions of nanoelectronic devices into high density system requires skillful architecture and reasonable layout. Interconnection is the most basic element of this architecture. The key for interconnect is to coordinate the input/output in the system. Advanced computing needs to store ton of data in a tiny physical space and use and process them very quickly. This requires a special structure to control and coordinate the various components.

\section{Acknowledgements}

This work was supported by National High Technology Research and Development Program of China
(No. 2011AA050504), Shanghai Science and Technology Grant (No. 12nm0503800 and No. 12nm0503500), and the Analytical and Testing Center of SJTU.

\section{References}

[1] Walt A. de Heer, A. Châtelain and D. Ugarte, "A carbon nanotube field-emission electron source", Science 270(5239), 1179-1180 (1995). http://dx.doi.org/10. 1126/science . 270.5239.1179

[2] R. H. Baughman, A. A. Zakhidov and W. A. De Heer, "Carbon nanotubes-the route toward applications", Science 297(5582), 787-792 (2002). http://dx. doi.org/10.1126/science. 1060928

[3] A. A. Kuznetzov, S. B. Lee, M. Zhang, R. H. Baughman and A. A. Zakhidov, "Electron field emission from transparent multiwalled carbon nanotube sheets for inverted field emission displays", Carbon 48(1), 41-46 (2010). http://dx.doi.org/10.1016/j. carbon. 2009.08.009

[4] M. K. Shin, J. Oh, M. Lima, M. E. Kozlov, S. J. Kim and R. H. "Baughman, elastomeric conductive composites based on carbon nanotube forests", Adv. Mater. 22(24), 2663-2667 (2010). http://dx.doi.org/ 10. 1002 /adma. 200904270

[5] M. Monti, M. Natali, R. Petrucci, J M. Kenny and L. Torre, "Impact damage sensing in glass fiber reinforced composites based on carbon nanotubes by electrical resistance measurements", J. Appl. Polym. Sci. 122(4), 2829-2836 (2011). http://dx.doi.org/10.1002/app. 34412

[6] A. Aviram and M. A. Ratner, "Molecular rectifiers", Chem. Phys. Lett. 29(2), 277-283 (1974). http://dx. doi.org/10.1016/0009-2614(74)85031-1

[7] J. M. Tour, R. Wu and J. S. Schumm, "Approaches to orthogonally fused conducting polymers for molecular electronics", J. Am. Chem. Soc. 112(14), 5662-5663 (1990). http://dx.doi.org/10.1021/ja00170a053

[8] J. M. Tour, L. Jones, D. L. Pearson, J. J. S. Lamba, T. P. Burgin, G. M. Whitesides, D. L. Allara, A. N. Parikh and S. Atre, "Self-Assembled monolayers and multilayers of conjugated thiols, .alpha.,.omega.dithiols, and thioacetyl-containing adsorbates. Understanding attachments between potential molecular wires and gold surfaces", J. Am. Chem. Soc. 117(37), 9529-9534 (1995). http://dx.doi.org/10. $1021 / \mathrm{ja} 00142 \mathrm{a} 021$

[9] J. J. Hopfield, J. N. Onuchic and D. N. Beratan, "A molecular shift register based on electron transfer", Science 241(4867), 817-820 (1988). http://dx.doi. org/10.1126/science. 241.4867.817

[10] M. H. Tsai, T. H. Lu and Y. H. Tang, "The electronic and transport properties of a molecular junction studied by an integrated piecewise thermal equilibrium approach", J. Appl. Phys. 104(4), 043703 (2008). http://dx.doi.org/10.1063/1.2970164

[11] P. W. Fowler, B. T. Pickup and T. Z. Todorova, "Equiconducting molecular conductors", Chem. Phys. 
Lett. 465(1-3), 142-146 (2008). http://dx.doi.org/ $10.1016 / \mathrm{j} . \mathrm{cplett} .2008 .09 .048$

[12] T. Hansen, V. Mujica and M. A. Ratner, "Cotunneling model for current-induced events in molecular wires", Nano Lett. 8(10), 3525-3531 (2008). http://dx.doi. org/10.1021/nl801001q

[13] J. Kong, N. R. Franklin, C. Zhou, M. G. Chapline, S. Peng, K. Cho and H. J. Dai, "Nanotube molecular wires as chemical sensors", Science 287(5453), 622625 (2000). http://dx.doi.org/10.1126/science. 287.5453 .622

[14] N. R. Franklin, Y. Li, R. J. Chen, A. Javey and H. J. Dai, "Patterned growth of single-walled carbon nanotubes on full 4-inch wafers", Appl. Phys. Lett. 79(27), 4571-4573 (2001). http://dx.doi.org/ 10.1063/1. 1429294

[15] C. P. Collier, E. W. Wong, M. Belohradský, F. M. Raymo, J. F. Stoddart, P. J. Kuekes, R. S. Williams and J. R. Heath, "Electronically configurable molecular-based lLogic gates", Science 285(5426), 391394 (1999). http://dx.doi.org/10.1126/science. 285.5426. 391

[16] D. I. Gittins, D. Bethell, D. J. Schiffrin and R. J. Nichols, "A nanometre-scale electronic switch consisting of a metal cluster and redox-addressable groups", Nature 408(6808), 67-69 (2000). http://dx.doi.org/ $10.1038 / 35040518$

[17] S. Katano, Y. Kim, M. Hori, M. Trenary and M. Kawai, "Reversible control of hydrogenation of a single molecule", Science 316(5833), 1883-1886 (2007). http://dx.doi.org/10.1126/science. 1141410

[18] R. M. Metzger, B. Chen, U. Hopfner, M. V. Lakshmikantham, D. Vuillaume, T. Kawai, X. L. Wu, H. Tachibana, T. V. Hughes, H. Sakurai, J. W. Baldwin, C. Hosch, M. P. Cava, L. Brehmer and G. J. Ashwell, "Unimolecular electrical rectification in hexadecylquinolinium tricyanoquinodimethanide", J. Am. Chem. Soc. 119(43), 10455-10466 (1997). http://dx. doi.org/10.1021/ja971811e

[19] B. Chen and R. M. Metzger, "Rectification between 370 and $105 \mathrm{~K}$ in hexadecylquinolinium tricyanoquinodimethanide", J. Phys. Chem. B 103(21), 4447-4451 (1999). http://dx.doi.org/10.1021/jp990006e

[20] J. Zhao, C. Zeng, X. Cheng, K. D. Wang, G. W. Wang, J. L. Yang, J. G. Hou and Q. S. Zhu, "Single C[sub 59]N Molecule as a molecular rectifier", Phys. Rev. Lett. 95(4), 045502/1-4 (2005). http://dx.doi.org/ 10.1103/PhysRevLett. 95.045502

[21] Y. Wada, T. Uda, M. Lutwyche, S. Kondo and S. Heike, "A proposal of nanoscale devices based on atom/molecule switching", J. Appl. Phys. 74(12), 7321-7328 (1993). http://dx.doi.org/10.1063/1. 354999

[22] D. Goldhaber-gordon, M. S. Montemerlo, J. C. Love, G. J. Opiteck, James and J. C. Ellenbogen, "Overview of nanoelectronic devices", Proceedings of the IEEE 85(4), 521-540 (1997). http://dx.doi.org/10.1109/ 5.573739
[23] C. Joachim, J. K. Gimzewski, R. R. Schlittler and C. Chavy, "Electronic transparence of a single $\mathrm{C}_{60}$ molecule", Phys. Rev. Lett. 74(11), 2102-2105 (1995). http://dx.doi.org/10.1103/PhysRevLett.74.2102

[24] C. Joachim and J. K. Gimzewski, "An electromechanical amplifier using a single molecule", Chemical Physics Letters 265(3-5), 353-357 (1997). http://dx. doi .org/10.1016/S0009-2614 (97)00014-6

[25] C. Joachim, J. K. Gimzewski and H. Tang, "Physical principles of the single- $\mathrm{C}_{60}$ transistor effect", Phys. Rev. B-Condensed Matter and Materials Physics 58(24), 16407-16417 (1998). http://dx.doi.org/10. 1103/PhysRevB.58.16407

[26] Y. Wada, "Atom electronics: a proposal of atom/molecule switching devices", Annals of the New York Academy of Sciences 852(1), 257-276 (1998). http://dx.doi.org/10.1111/j.1749-6632. 1998.tb09878.x

[27] J. F. Mennemann, A. Jüngel and H. Kosina, "Transient schrödinger-poisson simulations of a high-frequency resonant tunneling diode oscillator", J. Comput. Phys. 239, 187-205 (2013). http://dx.doi.org/10.1016/j. jcp. 2012.12.009

[28] G. Q. Zhang, S. Finefrock, D. X. Liang, Gautam, G. Yadav, H. R. Yang, H. Y. Fang and Y. Wu, "Semiconductor nanostructure -based photovoltaic solar cells", Nanoscale 3, 2430-2443 (2011). http://dx.doi.org/ $10.1039 / \mathrm{c} 1 \mathrm{nr} 10152 \mathrm{~h}$

[29] Moore, "Cramming more components onto integrated circuits", Proc. IEEE 86(1), 82-85 (1998). http://dx. doi.org/10.1109/JPROC. 1998.658762

[30] M. Lundstrom and Z. Ren, "Essential physics of carrier transport in nanoscale MOSFETs", IEEE Trans. Electron Devices 49(1), 133 (2002). http://dx.doi. org/10.1109/16.974760

[31] B. Winstead and U. Ravaioli, "Simulation of schottky barrier MOSFET's with a coupled quantum injection/monte carlo technique", IEEE Trans. Electron Devices 47(6), 1241 (2000). http://dx.doi.org/10. $1109 / 16.842968$

[32] G. Baccarani and S. Reggiani, "A compact double-gate MOSFET model comprising quantum-mechanical and nonstatic effects", IEEE Trans. Electron Devices 46(8), 1656 (1999). http://dx.doi.org/10.1109/16.777154

[33] T. Dürkop, S. A. Getty, Enrique Cobas and M. S. Fuhrer, "Extraordinary mobility in semiconducting carbon nanotubes", Nano Lett. 4(1), 35-39 (2004). http://dx.doi.org/10.1021/nl034841q

[34] C. W. Zhou, J. Kong and H. J. Dai, "Electrical measurements of individual semiconducting single-wall carbon nanotubes of warious diameters", Appl. Phys. Lett. 76(12), 1597 (2000). http://dx.doi.org/10. 1063/1.126107

[35] A. Bachtold, P. Hadley, T. Nakanishi and C. Dekker, "Logic circuits with carbon nanotube transistors", Science 294(5545), 1317-1320 (2001). http://dx.doi. org/10.1126/science. 1065824

[36] S. J. Wind, J. Appenzeller, R. Martel, V. Derycke and $\mathrm{Ph}$. Avouris, "Vertical scaling of carbon nanotube 
field-effect transistors using top gate electrodes", Appl. Phys. Lett. 80(20), 3817 (2002). http://dx.doi.org/ 10.1063/1.1480877

[37] M. H. Yang, K. B. K. Teo, L. Gangloff, W. I. Milne, D. G. Hasko, Y. Robert and P. Legagneux, "Advantages of top-gate, high-k dielectric carbon nanotube field-effect transistors", Appl. Phys. Lett. 88(11), 113 507 (2006). http://dx.doi.org/10.1063/1.2186100

[38] S. H. Hur, M. H. Yoon, A. Gaur, M. Shim, A. Facchetti, T. J. Marks and J. A. Rogers, "Organic nanodielectrics for low voltage carbon nanotube thin film transistors and complementary logic gates", J. Am. Chem. Soc. 127(40), 13808 (2005). http://dx.doi. org/10.1021/ja0553203

[39] R. Martel, T. Schmidt, H. R. Shea, T. Hertel and $\mathrm{Ph}$. Avouris, "Single- and multi-wall carbon nanotube field-effect transistors", Appl. Phys. Lett. 73(17), 2447 (1998). http://dx.doi.org/10.1063/1.122477

[40] S. J. Tans, A. R. M. Verschueren and C. Dekker, "Room-temperature transistor based on a single carbon nanotube", Nature 393(6680), 49-52 (1998). http://dx.doi.org/10.1038/29954

[41] C. X. Chen and Y. F. Zhang, "Carbon nanotube multichanneled field-effect transistors", J. Nanosci. Nanotech. 6(12), 3789-3793 (2006). http://dx.doi.org/ 10.1166/jnn.2006.626

[42] M. M. Shulaker, G. Hills, N. Patil, H. Wei, H. Y. Chen, H. S. P. Wong and S. Mitra, "Carbon nanotube computer", Nature 501(7468), 526-530 (2013). http://dx. doi.org/10.1038/nature12502

[43] J. W. Fergus, "Solid electrolyte based sensors for the measurement of CO and hydrocarbon gases", Sens. Actuators B-Chem 122(2), 683-693 (2007). http://dx. doi.org/10.1016/j.snb.2006.06.024

[44] R. Ramamoorthy, P. K. Dutta and S. A. Akbar, "Oxygen sensors: materials, methods, designs and applications", J. Mater. Sci. 38(21), 4271-4282 (2003). http://dx.doi.org/10.1023/A: 1026370729205

[45] D. D. Lee, D. S. Lee, "Environmental gas sensors", IEEE Sens. J. 1, 214-224 (2001). http://dx.doi.org/ 10.1109/JSEN . 2001.954834

[46] A. M. Azad, S. A. Akbar, S. G. Mhaisalkar, L. D. Birkefeld and K. S. Goto, "Solid-state gas sensors: A Review", J. Electrochem. Soc. 139(12), 3690-3704 (1992). http://dx.doi.org/10.1149/1.2069145

[47] J. Janata, M. Josowicz, P. Vanysek, D. M. DeVaney, "Chemical sensors", Anal. Chem. 70(20), 179-208 (1998). http://dx.doi.org/10.1021/a1980010w

[48] R. Knake, P. Jacquinot, A. W. E. Hodgson and P. C. Hauser, "Amperometric sensing in the gas-phase", Anal. Chim. Acta 549(1-2), 1-9 (2005). http://dx. doi.org/10.1016/j.aca.2005.06.007

[49] J. Wang, "Carbon-nanotube based electrochemical biosensors: A Review", Electroanalysis 17(1), 7-14 (2005). http://dx.doi.org/10.1002/elan. 200403113

[50] M. Valcárcel, S. Cárdenas and B. M. Simonet, "Role of carbon nanotubes in analytical science", Anal.
Chem 79(13), 4788-4797 (2007). http://dx.doi.org/ 10.1021/ac070196m

[51] G. Gruner, "Carbon nanotube transistors for biosensing applications", Anal. Bioanal. Chem. 384(2), 322-335 (2006). http://dx.doi.org/10.1007/ s00216-005-3400-4

[52] B. L. Allen, P. D. Kichambare and A. Star, "Carbon nanotube field-effect-transistor-based biosensors", Adv. Mater. 19(11), 1439-1451 (2007). http://dx. doi.org/10.1002/adma. 200602043

[53] T. Zhang, S. Mubeen, N. V. Myung and M. A. Deshusses, "Recent progress in carbon nanotubebased gas sensors", Nanotechnology 19(33), 332001 (2008). http://dx.doi.org/10.1088/0957-4484/19/ $33 / 332001$

[54] S. J. Tans, A. R. M. Verschueren and Cees Dekker, "Room-temperature transistor based on a single carbon nanotube", Nature 393(6680), 49-52 (1998). http://dx.doi.org/10.1038/29954

[55] R. Martel, T. Schmidt, H. R. Shea, T. Hertel and Ph. Avouris, "Single- and multi-wall carbon nanotube field-effect transistors", Appl. Phys. Lett. 73(17), 2447 (1998). http://dx.doi.org/10.1063/1.122477

[56] J. Kong, N. R. Franklin, C. W. Zhou, M. G. Chapline, S. Peng, K. Cho, H. J. Dai, "Nanotube molecular wires as chemical sensors", Science 287(5453), 622625 (2000). http://dx.doi.org/10.1126/science. 287.5453 .622

[57] J. Koh, B. Kim, S. Hong, H. Choi and H. Im, "Nanotube-based chemical and biomolecular sensors", J. Mater. Sci. Technol. 24(4), 578-588 (2008). http: // WWw. jmst . org/EN/Y2008/V24/I04/578

[58] S. Chattopadhyay, A. Ganguly, K. H. Chen and L. C. Chen, "One-dimensional group III-Nitrides: growth, properties, and applications in nanosensing and nano-optoelectronics", Crit. Rev. Solid State Mater. Sci. 34(3-4), 224-279 (2009). http://dx.doi. org/10.1080/10408430903352082

[59] A. Qureshi, W. P. Kang, J. L. Davidson and Y. Gurbuz, "Review on carbon-derived, solid-state, micro and nano sensors for electrochemical sensing applications", Diam. Relat. Mater. 18(12), 1401-1420 (2009). http://dx.doi.org/10.1016/j. diamond. 2009.09.008

[60] S. J. Pearton, F. Ren, Y. L. Wang, B. H. Chu, K. H. Chen, C. Y. Chang, W. Lim, J. Lin and D. P. Norton, "Recent advances in wide bandgap semiconductor biological and gas sensors", Prog. Mater. Sci. 55(1), 159 (2010). http://dx.doi.org/10.1016/j.pmatsci. 2009.08.003

[61] Y. Zhang, J. H. Liu and C. C. Zhu, "Novel gas ionization sensors using carbon nanotubes", Sens. Lett. 8(2), 219-227 (2010). http://dx.doi.org/10.1166/ sl.2010.1270

[62] P. A. Smith, C. J. Lepage, K. L. Harrer and P. J. Brochu, "Hand-held photoionization instruments for quantitative detection of sarin vapor and for rapid qualitative screening of contaminated objects", J. Oc- 
cup. Environ. Hyg. 4(10), 729-738 (2007). http://dx. doi.org/10.1080/15459620701547233

[63] Y. W. Cheng, Z. Yang, H. Wei, Y. Y. Wang, L. M. Wei and Y. F. Zhang, "Progress in carbon nanotube gas sensor research", Acta Phys. Chim. Sin. 26(12), 3127-3142 (2010). http://dx.doi.org/10.3866/PKU. WHXB20101138

[64] Z. Y. Hou, H. Liu, X. Wei, J. H. Wu, W. M. Zhou, Y. F. Zhang, D. Xu and B. C. Cai, "MEMS-based microelectrode system incorporating carbon nanotubes for ionization gas sensing", Sens. Actuators B: Chemical 127(2), 637-648 (2007). http://dx.doi.org/10.1016/ j.snb.2007.05.026

[65] N. Dattoli, Q. Wan, W. Guo, Y. Chen, X. Pan and W. Lu, "Fully transparent thin-film transistor devices based on $\mathrm{SnO}_{2}$ nanowires", Nano Lett. 7(8), 2463-2469 (2007). http://dx.doi.org/10.1021/nl0712217

[66] C. Korman and GE Global Research, "Overview of solar photovoltaic markets and technology", Presentation given for MSE 542, A short course presented at Cornell University, March 28 (2006).

[67] G. Q. Zhang, S. Finefrock, D. X. Liang, G. G. Yadav, H. R. Yang, H. Y. Fang and Y. Wu, "Semiconductor nanostructure-based photovoltaic solar cells", Nanoscale 3(6), 2430-2443 (2011). http://dx.doi. org/10.1039/c1nr10152h

[68] R. D. Schaller and V. I. Klimov, "High efficiency carrier multiplication in PbSe nanocrystals: implications for solar energy conversion", Phys. Rev. Lett. 92(18), 186601-186604 (2004). http://dx.doi.org/10.1103/ PhysRevLett.92.186601

[69] R. J. Ellingson, M. C. Beard, J. C. Johnson, P. Yu, O. I. Micic, A. J. Nozik, A. Shabaev and A. L. Efros, "Highly efficient multiple exciton generation in colloidal PbSe and PbS quantum dots", Nano Lett. 5(5), 865-871 (2005). http://dx.doi.org/10.1021/ nl0502672

[70] J. E. Murphy, M. C. Beard, A. G. Norman, S. P. Ahrenkiel, J. C. Johnson, P. Yu, O. I. Mićić, R. J. Ellingson and A. J. Nozik, "PbTe colloidal nanocrystals: synthesis, characterization, and multiple exciton generation", J. Am. Chem. Soc. 128(10), 3241-3247 (2006). http://dx.doi.org/10.1021/ja0574973

[71] R. D. Schaller, M. Sykora, S. Jeong and V. I. Klimov, "High-efficiency carrier multiplication and ultrafast charge separation in semiconductor nanocrystals studied via time-resolved photoluminescence", J. Phys. Chem. B. 110(50), 25332-25338 (2006). http://dx . doi.org/10.1021/jp065282p

[72] M. C. Beard, K. P. Knutsen, P. Yu, J. M. Luther, Q. Song, W. K. Metzger, R. J. Ellingson and A. J. Nozik, "Multiple exciton generation in colloidal silicon nanocrystals", Nano Lett. 7(8), 2506-2512 (2007). http://dx.doi.org/10.1021/n10714861
[73] J. J. H. Pijpers, E. Hendry, M. T. W. Milder, R. Fanciulli, J. Savolainen, J. L. Herek, D. Vanmaekelbergh, S. Ruhman, D. Mocatta, D. Oron, A. Aharoni, U. Banin and M. Bonn, "Carrier multiplication and Its reduction by photodoping in colloidal InAs quantum dots", J. Phys. Chem. C. 111(11), 4146-4152 (2007). http:// dx.doi.org/10.1021/jp066709v

[74] A. J. Nozik, "Quantum dot solar cells", Physica E 14(1-2), 115-120 (2002). http://dx.doi.org/10. 1016/S1386-9477 (02)00374-0

[75] G. F. Brown and J. Wu, "Third generation photovoltaics", Laser Photonics Rev. 3(4), 394-405 (2009). http://dx.doi.org/10.1002/lpor.200810039

[76] R. Zhang, X. Y. Chen, J. J. Lu and W. Z. Shen, "Photocurrent of hydrogenated nanocrystalline silicon thin film/crystalline silicon heterostructure", J. Appl. Phys. 102(12), 123708 (2007). http://dx.doi.org/ $10.1063 / 1.2826742$

[77] C. Chen, Y. Lu, E. S. Kong, Y. Zhang and S. T. Lee, "Nanowelded carbon-nanotube-based solar microcells", Small 4(9), 1313-1318 (2008). http://dx.doi. org/10.1002/smll.200701309

[78] N. M. Gabor, Z. Zhong, K. Bosnick, J. Park and P. L. McEuen, "Extremely efficient multiple electronhole pair generation in carbon nanotube photodiodes", Science 325(5946), 1367-1371 (2009). http://dx.doi. org/10.1126/science.1176112

[79] M. Albota, D. Beljonne, J. L. Brédas, J. E. Ehrlich, J. Y. Fu, A. A. Heikal, S. E. Hess, T. Kogej, M. D. Levin, S. R. Marder, D. M. Maughon, J. W. Perry, H. Röckel, M. Rumi, G. Subramaniam, W. W. Webb, X. $\mathrm{L}$. $\mathrm{Wu}$ and $\mathrm{C} . \mathrm{Xu}$, "Design of organic molecules with large two-photon absorption cross sections", Science 281(5383), 1653-1656 (1998). http://dx.doi.org/10. 1126/science. 281.5383.1653

[80] C. Strumpel, M. McCann, G. Beaucarne, V. Arkhipov, A. Slaoui, V. Svrcek, C. del Canizo and I. Tobias, "Modifying the solar spectrum to enhance silicon solar cell efficiency-An overview of available materials", Sol. Energy Mater. Sol. Cells 91(4), 238-249 (2007). http://dx.doi.org/10.1016/j.solmat.2006.09.003

[81] E. Garnett and P. D. Yang, "Light trapping in silicon nanowire solar cells", Nano Lett. 10(3), 1082-1087 (2010). http://dx.doi.org/10.1021/nl100161z

[82] H. J. Hovel, "Semiconductors and semimetals", Academic Press, New York, San Francisco, London Subsidiary of Harcoun Brace Jvanovich Publishers 48-69 (1976).

[83] F. Pehnchon and P. Mialhe, "Toward a theoretical limit of solar cell efficiency with light trapping and sub-structure", Solar Energy 54(6), 381-385 (1995). http://dx.doi.org/10.1016/ 0038-092X (95) 00005-C 